\title{
Novel 2,4,6-Trimethylbenzenesulfonyl Hydrazones with Antibacterial Activity: Synthesis and In Vitro Study
}

\author{
Lukasz Popiołek $^{1, *}$, Sylwia Szeremeta ${ }^{1}$, Anna Biernasiuk ${ }^{2}$ (D) and Monika Wujec ${ }^{1}$ (D) \\ 1 Department of Organic Chemistry, Faculty of Pharmacy, Medical University of Lublin, 4A Chodźki Street, \\ 20-093 Lublin, Poland; sszeremeta96@o2.pl (S.S.); monika.wujec@umlub.pl (M.W.) \\ 2 Department of Pharmaceutical Microbiology, Faculty of Pharmacy, Medical University of Lublin, \\ 1 Chodźki Street, 20-093 Lublin, Poland; anna.biernasiuk@umlub.pl \\ * Correspondence: lukasz.popiolek@umlub.pl; Tel.: +48-814-487-243
}

Citation: Popiołek, Ł.; Szeremeta, S.; Biernasiuk, A.; Wujec, M. Novel 2,4,6-Trimethylbenzenesulfonyl Hydrazones with Antibacterial Activity: Synthesis and In Vitro Study. Materials 2021, 14, 2723. https:// doi.org/10.3390/ma14112723

Academic Editors: Liliana Mazur and Katarzyna Jarzembska

Received: 30 March 2021

Accepted: 12 May 2021

Published: 21 May 2021

Publisher's Note: MDPI stays neutral with regard to jurisdictional claims in published maps and institutional affiliations.

Copyright: (c) 2021 by the authors. Licensee MDPI, Basel, Switzerland. This article is an open access article distributed under the terms and conditions of the Creative Commons Attribution (CC BY) license (https:// creativecommons.org/licenses/by/ $4.0 /)$.

\begin{abstract}
This research describes the synthesis and in vitro antimicrobial activity study of a series of 2,4,6-trimethylbenzenesulfonyl hydrazones. Twenty-five hydrazones (2-26) were synthesized on the basis of condensation reaction. The in vitro bioactivity study confirmed the potential application of obtained derivatives as antimicrobial agents. Among the tested compounds, the highest activity was discovered for derivative 24 , which possessed minimal inhibitory concentration (MIC) ranging from 7.81 to $15.62 \mu \mathrm{g} / \mathrm{mL}$ against Gram-positive reference bacterial strains. Synthesized benzenesulfonyl hydrazones can be applied as potential ligands for the synthesis of bioactive metal complexes.
\end{abstract}

Keywords: benzenesulfonyl hydrazones; bioactivity; antibacterial activity; MIC; MBC; ligands

\section{Introduction}

In recent years, the problem of the emerging resistance of bacteria and fungi to antibiotics and chemotherapeutic agents has been observed [1]. Even 30-40 years ago, this problem was not so noticeable because from the 1940s to the 1980s, the pharmaceutical industry introduced many classes of medicines to the treatment [1-3]. In the fight against bacterial and fungal infections, antimicrobial agents are used, which can be divided into two main classes: obtained by isolating from naturally occurring organisms in the ecosystem and by chemical modification of them, namely, antibiotics, and obtained by chemical synthesis, namely, chemotherapeutic agents. Recent years have shown that antimicrobial agents that have been used for decades are not always effective in treating infections [1-3]. Due to these factors, scientists are seeking new molecules with antimicrobial properties. The discovery of new medicines with antimicrobial activity profile could eliminate infections that the current healthcare system cannot cope with, and it would also be possible to shorten hospitalization time and possible complications, as well as to reduce the number of therapeutic agents used [1-3].

One class of compounds that has attracted the attention of scientists in recent years is benzenesulfonohydrazide derivatives. This interest results from the possibility of their wide use in chemical synthesis as intermediates [4-7], as well as the fact that this group of compounds has significant and wide spectrum of biological properties, including anticancer [8-12], antibacterial [13-17], antifungal [14,18-21], and antidepressant activity [22,23].

Benzenesulfonyl hydrazones also play an important role in coordination chemistry as ligands in the synthesis of metal complexes [24-31]. This is due to the fact that they possess a number of possible metal binging sites and can act as both bi- and tetradentate ligands, depending on the portion of the ligand involved in the metal complexation [28]. According to scientific reports, such complexes display interesting biological activity. They possess mainly antibacterial [24-28], anticancer [29], and antimalarial properties [30]. Aslan et al. reported the synthesis of novel benzenesulfonic acid derivatives and its $\mathrm{Ni}(\mathrm{II}), \mathrm{Pd}(\mathrm{II})$, $\mathrm{Pt}(\mathrm{II}), \mathrm{Cu}(\mathrm{II})$, and Co(II) complexes (Figure 1A) [24]. Synthesized compounds showed 
potent inhibition against tested bacterial strains [24]. Similar complexes with significant antibacterial activity were also synthesized by Özdemir et al. (Figure 1B) [25] and Özbek et al. (Figure 1C) [27].

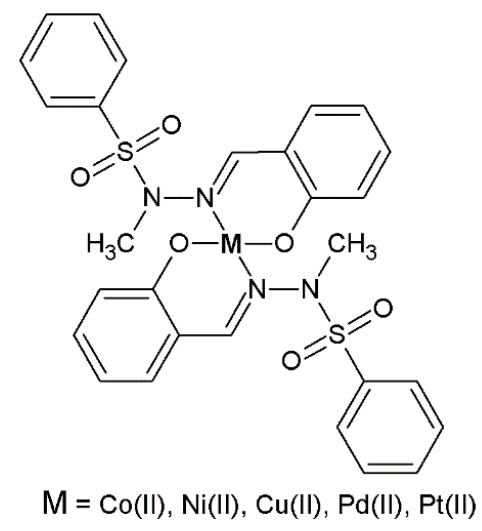

A<smiles>[R]c1c2on(NS(C)(=O)=O)c([R])c(NNS(C)(=O)=O)n-2oc2ccc(C)cc12</smiles>

$\mathrm{M}=\mathrm{Ni}(\mathrm{II}), \mathrm{Co}(\mathrm{II}) ; \mathrm{R}=\mathrm{H}, \mathrm{CH}_{3}$

B

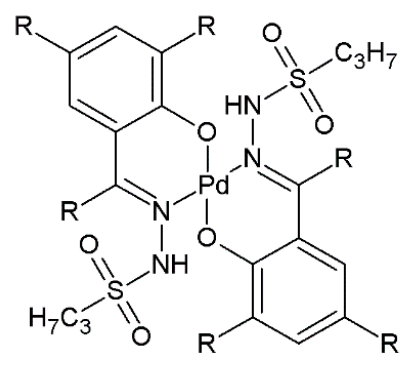

C

Figure 1. Benzenesulfonyl hydrazone complexes with antibacterial activity $(\mathbf{A}-\mathbf{C})$.

In the scientific literature findings, there is also some information that concerns the synthesis and activity of organometallic-based sulfonyl hydrazones [31]. Concha et al. synthesized series of organometallic tosylhydrazones containing cyrhetrenyl and cymantrenyl moieties and evaluated them for potential antitubercular and antitumor activity [31].

Prompted by the above-mentioned fact, in this research, we designed, synthesized, and tested for in vitro antimicrobial activity a novel series of 2,4,6-trimethylbenzenesulfonyl hydrazones with the aim to obtain promising potential antimicrobial agents that can be used further as potential ligands for the synthesis of bioactive metal complexes.

\section{Materials and Methods}

\subsection{Chemistry}

All reagents and solvents used in this research were purchased from Sigma-Aldrich (Munich, Germany) and Merck Co. (Darmstadt, Germany) and used without further purification. Thin-layer chromatography (TLC) on plates covered with silica gel (aluminum oxide 60 F-254, Merck Co., Kenilworth, NJ, USA) was used to check the purity of the obtained compounds and to monitor the progress of the reaction. Chloroform-ethanol mixture in the 10:1 $(v / v)$ ratio was used as the mobile phase. The spots were detected by irradiation with UV light at a wavelength of $\lambda=254 \mathrm{~nm} .{ }^{1} \mathrm{H}$ NMR and ${ }^{13} \mathrm{C}$ NMR spectra were recorded on the Bruker Avance 300 and 600 apparatus (Bruker BioSpin GmbH, Rheinstetten, Germany). The melting points of the obtained compounds were measured with a Fisher-Johns apparatus (Fisher Scientific, Schwerte, Germany) and presented without any correction. The elemental analysis was determined by the Perkin Elmer 2400 series II CHNS/O analyzer (Waltham, MA, USA), and the results were within $\pm 0.4 \%$ of the theoretical value. 


\subsubsection{Preparation of 2,4,6-Trimethylbenzenesulfonyl Hydrazones (2-26)}

The 2,4,6-trimethylbenzenesulfonyl hydrazones were synthesized with the use of the method described earlier by our group for the synthesis of hydrazide-hydrazones [32-34].

We dissolved 0.01 mole of 2,4,6-trimethylbenzenesulfonohydrazide (1) in ethanol ( $5 \mathrm{~mL}, 96 \%$ ). Then, 0.011 mole of appropriate substituted benzaldehyde was added, and the mixture was heated under reflux for $3 \mathrm{~h}$. The substituted benzaldehydes used in this research are presented in Scheme 1. After that, the solution was cooled to room temperature and placed in the refrigerator for $24 \mathrm{~h}$. Subsequently the precipitate formed was filtered off and re-crystallized from ethanol (96\%).

\subsubsection{Physicochemical Properties of 2,4,6-Trimethylbenzenesulfonyl Hydrazones (2-26)}

$\mathrm{N}$-[(3-Ethoxy-2-hydroxyphenyl)methylidene]-2,4,6-trimethylbenzenesulfonohydrazide (2)

Yellow powder; CAS Number: 1799087-18-5; M.p.: $132{ }^{\circ} \mathrm{C}$; Yield: $52 \%$; ${ }^{1} \mathrm{H}$ NMR (300 MHz, DMSO- $\left.d_{6}\right) \delta(\mathrm{ppm}): 1.29-1.31\left(\mathrm{t}, 3 \mathrm{H}, \mathrm{CH}_{3}, J=9 \mathrm{~Hz}, J=6 \mathrm{~Hz}\right), 2.24\left(\mathrm{~s}, 3 \mathrm{H}, \mathrm{CH}_{3}\right)$, $2.62\left(\mathrm{~s}, 6 \mathrm{H}, 2 \times \mathrm{CH}_{3}\right), 3.98-4.05\left(\mathrm{q}, 2 \mathrm{H}, \mathrm{CH}_{2}, J=6 \mathrm{~Hz}\right), 6.71-6.76(\mathrm{t}, 1 \mathrm{H}, \mathrm{ArH}, J=9 \mathrm{~Hz}, J=6$ $\mathrm{Hz}), 6.86-6.92(\mathrm{~m}, 1 \mathrm{H}, \mathrm{ArH}), 6.94-7.00(\mathrm{~m}, 1 \mathrm{H}, \mathrm{ArH}), 7.05(\mathrm{~s}, 2 \mathrm{H}, \mathrm{ArH}), 8.20(\mathrm{~s}, 1 \mathrm{H},=\mathrm{CH})$, $8.98(\mathrm{~s}, 1 \mathrm{H}, \mathrm{OH}), 11.54(\mathrm{~s}, 1 \mathrm{H}, \mathrm{NH}) ;{ }^{13} \mathrm{C}$ NMR $\left(75 \mathrm{MHz}, \mathrm{DMSO}-d_{6}\right) \delta(\mathrm{ppm}): 15.18\left(\mathrm{CH}_{3}\right)$, $21.63\left(2 \times \mathrm{CH}_{3}\right), 23.14\left(\mathrm{CH}_{3}\right), 64.52\left(\mathrm{CH}_{2}\right), 118.85,120.19,132.16,133.49,139.64,142.86$, $146.53,147.45\left(10 \times \mathrm{C}_{\mathrm{ar}}\right), 147.54(=\mathrm{CH}), 149.22,163.38\left(2 \times \mathrm{C}_{\mathrm{ar}}\right)$.

$N$-[(3,4-Dimethoxyphenyl)methylidene]-2,4,6-trimethylbenzenesulfonohydrazide (3)

Yellowish powder; CAS Number: $1799143-26-2$; M.p.: $140{ }^{\circ} \mathrm{C}$; Yield: $61 \% ;{ }^{1} \mathrm{H}$ NMR $\left(300 \mathrm{MHz}, \mathrm{DMSO}-d_{6}\right) \delta(\mathrm{ppm}): 2.23\left(\mathrm{~s}, 3 \mathrm{H}, \mathrm{CH}_{3}\right), 2.65\left(\mathrm{~s}, 6 \mathrm{H}, 2 \times \mathrm{CH}_{3}\right), 3.72\left(\mathrm{~s}, 3 \mathrm{H}, \mathrm{OCH}_{3}\right)$, $3.75\left(\mathrm{~s}, 3 \mathrm{H}, \mathrm{OCH}_{3}\right), 6.93-6.95(\mathrm{~d}, 1 \mathrm{H}, \mathrm{ArH}, J=6 \mathrm{~Hz}), 7.04(\mathrm{~s}, 2 \mathrm{H}, \mathrm{ArH}), 7.05-7.08(\mathrm{~m}, 1 \mathrm{H}$, $\mathrm{ArH}), 7.81(\mathrm{~s}, 1 \mathrm{H}, \mathrm{ArH}), 8.64(\mathrm{~s}, 1 \mathrm{H},=\mathrm{CH}), 11.38(\mathrm{~s}, 1 \mathrm{H}, \mathrm{NH}) ;{ }^{13} \mathrm{C}$ NMR $\left(75 \mathrm{MHz}\right.$, DMSO-d $\left.d_{6}\right)$ $\delta$ (ppm): $20.87\left(2 \times \mathrm{CH}_{3}\right), 23.23\left(\mathrm{CH}_{3}\right), 56.79\left(\mathrm{OCH}_{3}\right), 57.89\left(\mathrm{OCH}_{3}\right), 111.91,114.10,122.11$, $127.07,129.35,132.05,133.84,139.73\left(10 \times \mathrm{C}_{\mathrm{ar}}\right), 142.68(=\mathrm{CH}), 149.32,150.88\left(2 \times \mathrm{C}_{\mathrm{ar}}\right)$.

$\mathrm{N}$-[(3-Ethoxy-4-hydroxyphenyl)methylidene]-2,4,6-trimethylbenzenesulfonohydrazide (4)

Brown powder; CAS Number: $1799022-47-1$; M.p.: $142{ }^{\circ} \mathrm{C}$; Yield: $52 \%$; ${ }^{1} \mathrm{H}$ NMR (300 MHz, DMSO- $\left.d_{6}\right) \delta(\mathrm{ppm}): 1.30-1.32\left(\mathrm{t}, 3 \mathrm{H}, \mathrm{CH}_{3}\right), 2.23\left(\mathrm{~s}, 3 \mathrm{H}, \mathrm{CH}_{3}\right), 2.64(\mathrm{~s}, 6 \mathrm{H}, 2 \times$ $\left.\mathrm{CH}_{3}\right), 3.94-4.01\left(\mathrm{q}, 2 \mathrm{H}, \mathrm{CH}_{2}, J=9 \mathrm{~Hz}, J=6 \mathrm{~Hz}\right), 6.74-6.77(\mathrm{~d}, 1 \mathrm{H}, \mathrm{ArH}, J=9 \mathrm{~Hz}), 6.86-6.90$ $(\mathrm{m}, 1 \mathrm{H}, \mathrm{ArH}), 7.03(\mathrm{~s}, 2 \mathrm{H}, \mathrm{ArH}), 7.22-7.25(\mathrm{~d}, 1 \mathrm{H}, \mathrm{ArH}, J=9 \mathrm{~Hz}), 7.76(\mathrm{~s}, 1 \mathrm{H}, \mathrm{OH}), 8.55(\mathrm{~s}$, $1 \mathrm{H},=\mathrm{CH}), 11.26(\mathrm{~s}, 1 \mathrm{H}, \mathrm{NH}) ;{ }^{13} \mathrm{C}$ NMR $\left(75 \mathrm{MHz}, \mathrm{DMSO}-d_{6}\right) \delta(\mathrm{ppm}): 15.10\left(\mathrm{CH}_{3}\right), 20.87$ $\left(2 \times \mathrm{CH}_{3}\right), 23.24\left(\mathrm{CH}_{3}\right), 64.52\left(\mathrm{CH}_{2}\right), 115.92,125.74,125.88,132.03,133.88,139.71,142.62$, $146.26\left(10 \times \mathrm{C}_{\mathrm{ar}}\right), 147.57(=\mathrm{CH}), 149.35,150.57\left(2 \times \mathrm{C}_{\mathrm{ar}}\right)$.

$\mathrm{N}$-[(2,4-Dimethoxyphenyl)methylidene]-2,4,6-trimethylbenzenesulfonohydrazide (5)

Orange powder; CAS Number: 1799183-35-9; M.p.: $140{ }^{\circ} \mathrm{C}$; Yield: $45 \% ;{ }^{1} \mathrm{H}$ NMR $(300$ MHz, DMSO- $\left.d_{6}\right) \delta(\mathrm{ppm}): 2.23\left(\mathrm{~s}, 3 \mathrm{H}, \mathrm{CH}_{3}\right), 2.61\left(\mathrm{~s}, 6 \mathrm{H}, 2 \times \mathrm{CH}_{3}\right), 3.76\left(\mathrm{~s}, 3 \mathrm{H}, \mathrm{OCH}_{3}\right), 3.78$ $\left(\mathrm{s}, 3 \mathrm{H}, \mathrm{OCH}_{3}\right), 6.53-6.55(\mathrm{~m}, 2 \mathrm{H}, \mathrm{ArH}), 7.02(\mathrm{~s}, 2 \mathrm{H}, \mathrm{ArH}), 7.42-7.45(\mathrm{~d}, 1 \mathrm{H}, \mathrm{ArH}, J=9 \mathrm{~Hz})$, $8.13(\mathrm{~s}, 1 \mathrm{H},=\mathrm{CH}), 11.23(\mathrm{~s}, 1 \mathrm{H}, \mathrm{NH}) ;{ }^{13} \mathrm{C} \mathrm{NMR}\left(75 \mathrm{MHz}, \mathrm{DMSO}-d_{6}\right) \delta(\mathrm{ppm}): 20.86(2 \times$ $\left.\mathrm{CH}_{3}\right), 23.21\left(\mathrm{CH}_{3}\right), 56.04\left(\mathrm{OCH}_{3}\right), 56.79\left(\mathrm{OCH}_{3}\right), 98.66,106.87,115.09,126.39,132.04,133.90$, $139.63,141.62\left(10 \times \mathrm{C}_{\mathrm{ar}}\right), 142.58(=\mathrm{CH}), 159.17,162.70\left(2 \times \mathrm{C}_{\mathrm{ar}}\right)$.

$\mathrm{N}$-[(2,3-Dimethoxyphenyl)methylidene]-2,4,6-trimethylbenzenesulfonohydrazide (6)

White powder; CAS Number: $1799021-56-9$; M.p.: $118{ }^{\circ}$ C; Yield: $43 \% ;{ }^{1} \mathrm{H}$ NMR (300 MHz, DMSO- $\left.d_{6}\right) \delta(\mathrm{ppm}): 2.23\left(\mathrm{~s}, 3 \mathrm{H}, \mathrm{CH}_{3}\right), 2.63\left(\mathrm{~s}, 6 \mathrm{H}, 2 \times \mathrm{CH}_{3}\right), 3.70\left(\mathrm{~s}, 3 \mathrm{H}, \mathrm{OCH}_{3}\right), 3.79$ $\left(\mathrm{s}, 3 \mathrm{H}, \mathrm{OCH}_{3}\right), 7.03(\mathrm{~s}, 2 \mathrm{H}, \mathrm{ArH}), 7.04-7.12(\mathrm{~m}, 3 \mathrm{H}, \mathrm{ArH}), 8.17(\mathrm{~s}, 1 \mathrm{H},=\mathrm{CH}), 11.54(\mathrm{~s}, 1 \mathrm{H}$, $\mathrm{NH}) ;{ }^{13} \mathrm{C}$ NMR $\left(75 \mathrm{MHz}\right.$, DMSO-d 6 ) $\delta$ (ppm): $20.89\left(2 \times \mathrm{CH}_{3}\right), 23.20\left(\mathrm{CH}_{3}\right), 56.81\left(\mathrm{OCH}_{3}\right)$, $56.99\left(\mathrm{OCH}_{3}\right), 112.11,115.09,123.23,127.77,129.15,132.75,133.64,138.13\left(10 \times \mathrm{C}_{\mathrm{ar}}\right), 142.78$ $(=\mathrm{CH}), 150.31,151.98\left(2 \times \mathrm{C}_{\mathrm{ar}}\right)$.

$N$-[(2-Iodophenyl)methylidene]-2,4,6-trimethylbenzenesulfonohydrazide (7)

Bright yellow powder; M.p.: $170{ }^{\circ} \mathrm{C}$; Yield: $76 \% ;{ }^{1} \mathrm{H}$ NMR $\left(300 \mathrm{MHz}\right.$, DMSO- $\left.d_{6}\right) \delta$ (ppm): 2.24 (s, 3H, $\left.\mathrm{CH}_{3}\right), 2.63\left(\mathrm{~s}, 6 \mathrm{H}, 2 \times \mathrm{CH}_{3}\right), 7.05$ (s, 2H, ArH), 7.08-7.14 (m, 1H, ArH), 7.36-7.41 (t, $1 \mathrm{H}, \mathrm{ArH}, J=6 \mathrm{~Hz}, J=9 \mathrm{~Hz}), 7.53-7.56(\mathrm{~d}, 1 \mathrm{H}, \mathrm{ArH}, J=9 \mathrm{~Hz}), 7.85-7.88(\mathrm{~d}$, $1 \mathrm{H}, \mathrm{ArH}, J=9 \mathrm{~Hz}), 8.13(\mathrm{~s}, 1 \mathrm{H},=\mathrm{CH}), 11.87(\mathrm{~s}, 1 \mathrm{H}, \mathrm{NH}) ;{ }^{13} \mathrm{C} \mathrm{NMR}\left(75 \mathrm{MHz}, \mathrm{DMSO}-d_{6}\right) \delta$ 
(ppm): $20.89\left(\mathrm{CH}_{3}\right), 23.18\left(2 \times \mathrm{CH}_{3}\right), 100.15,126.57,129.07,132.08,132.17,133.70,135.67$, $139.60,140.15\left(11 \times \mathrm{C}_{\mathrm{ar}}\right), 142.91(=\mathrm{CH}), 148.51\left(\mathrm{C}_{\mathrm{ar}}\right)$.

$\mathrm{N}$-[(3-Iodophenyl)methylidene]-2,4,6-trimethylbenzenesulfonohydrazide (8)

Orange powder; M.p.: $128{ }^{\circ} \mathrm{C}$; Yield: 78\%; ${ }^{1} \mathrm{H}$ NMR $\left(300 \mathrm{MHz}, \mathrm{DMSO}-d_{6}\right) \delta(\mathrm{ppm})$ : $2.24\left(\mathrm{~s}, 3 \mathrm{H}, \mathrm{CH}_{3}\right), 2.63\left(\mathrm{~s}, 6 \mathrm{H}, 2 \times \mathrm{CH}_{3}\right), 7.05(\mathrm{~s}, 2 \mathrm{H}, \mathrm{ArH}), 7.15-7.20(\mathrm{t}, 1 \mathrm{H}, \mathrm{ArH}, J=9 \mathrm{~Hz}, J$ $=6 \mathrm{~Hz}), 7.49-7.51(\mathrm{~d}, 1 \mathrm{H}, \mathrm{ArH}, J=6 \mathrm{~Hz}), 7.69-7.72(\mathrm{~m}, 1 \mathrm{H}, \mathrm{ArH}), 7.86-7.90(\mathrm{~m}, 1 \mathrm{H}, \mathrm{ArH})$, $7.83(\mathrm{~s}, 1 \mathrm{H},=\mathrm{CH}), 11.76(\mathrm{~s}, 1 \mathrm{H}, \mathrm{NH}) ;{ }^{13} \mathrm{C}$ NMR $\left(75 \mathrm{MHz}, \mathrm{DMSO}-d_{6}\right) \delta(\mathrm{ppm}): 20.89\left(\mathrm{CH}_{3}\right)$, $23.16\left(2 \times \mathrm{CH}_{3}\right), 95.68,126.21,131.39,132.14,133.73,135.26,136.58,138.66,139.63(11 \times$ $\left.\mathrm{C}_{\mathrm{ar}}\right), 142.88(=\mathrm{CH}), 143.88\left(\mathrm{C}_{\mathrm{ar}}\right)$.

$\mathrm{N}$-[(4-Iodophenyl)methylidene]-2,4,6-trimethylbenzenesulfonohydrazide (9)

Light yellow powder; M.p.: $166{ }^{\circ} \mathrm{C}$; Yield: $46 \% ;{ }^{1} \mathrm{H}$ NMR $\left(300 \mathrm{MHz}\right.$, DMSO- $\left.d_{6}\right) \delta$ (ppm): $2.23\left(\mathrm{~s}, 3 \mathrm{H}, \mathrm{CH}_{3}\right), 2.62\left(\mathrm{~s}, 6 \mathrm{H}, 2 \times \mathrm{CH}_{3}\right), 7.04(\mathrm{~s}, 2 \mathrm{H}, \mathrm{ArH}), 7.26-7.29(\mathrm{~d}, 2 \mathrm{H}, \mathrm{ArH}, J$ $=9 \mathrm{~Hz}), 7.88-7.91(\mathrm{~d}, 2 \mathrm{H}, \mathrm{ArH}, J=9 \mathrm{~Hz}), 8.67(\mathrm{~s}, 1 \mathrm{H},=\mathrm{CH}), 11.68(\mathrm{~s}, 1 \mathrm{H}, \mathrm{NH}) ;{ }^{13} \mathrm{C} \mathrm{NMR}$ (75 MHz, DMSO- $\left.d_{6}\right) \delta(\mathrm{ppm}): 21.03\left(\mathrm{CH}_{3}\right), 23.19\left(2 \times \mathrm{CH}_{3}\right), 99.45,128.75,130.55,132.12$, $133.66,138.12,138.33,139.64\left(12 \times \mathrm{C}_{\mathrm{ar}}\right), 142.82(=\mathrm{CH})$.

$N$-[(2-Fluorophenyl)methylidene]-2,4,6-trimethylbenzenesulfonohydrazide (10)

Bright yellow powder; CAS Number: $1799182-76-5$; M.p.: $161^{\circ} \mathrm{C}$; Yield: $54 \% ;{ }^{1} \mathrm{H}$ NMR (300 MHz, DMSO- $\left.d_{6}\right) \delta(\mathrm{ppm}): 2.24\left(\mathrm{~s}, 3 \mathrm{H}, \mathrm{CH}_{3}\right), 2.63\left(\mathrm{~s}, 6 \mathrm{H}, 2 \times \mathrm{CH}_{3}\right), 7.05$ (s, 2H, $\left.\mathrm{ArH}\right)$, 7.19-7.27 (m, 1H, ArH), 7.35-7.45 (m, 1H, ArH), 7.55-7.61 (m, 1H, ArH), 7.85-7.88 (d, 1H, $\mathrm{ArH}, J=9 \mathrm{~Hz}), 8.09(\mathrm{~s}, 1 \mathrm{H},=\mathrm{CH}), 11.77(\mathrm{~s}, 1 \mathrm{H}, \mathrm{NH}) ;{ }^{13} \mathrm{C}$ NMR $\left(150 \mathrm{MHz}, \mathrm{DMSO}-d_{6}\right) \delta$ (ppm): $20.89\left(\mathrm{CH}_{3}\right), 23.15\left(2 \times \mathrm{CH}_{3}\right), 116.61,121.77,125.41,126.22,132.14,133.68,138.53$, $139.72\left(10 \times \mathrm{C}_{\mathrm{ar}}\right), 142.89(=\mathrm{CH}), 155.57,159.99\left(2 \times \mathrm{C}_{\mathrm{ar}}\right)$.

$\mathrm{N}$-[(3-Fluorophenyl)methylidene]-2,4,6-trimethylbenzenesulfonohydrazide (11)

Bright yellow powder; CAS Number: 1799086-95-5; M.p.: $126{ }^{\circ} \mathrm{C}$; Yield: $7 \%$; ${ }^{1} \mathrm{H}$ NMR $\left(300 \mathrm{MHz}, \mathrm{DMSO}-d_{6}\right) \delta(\mathrm{ppm}): 2.26\left(\mathrm{~s}, 3 \mathrm{H}, \mathrm{CH}_{3}\right), 2.62\left(\mathrm{~s}, 6 \mathrm{H}, 2 \times \mathrm{CH}_{3}\right), 7.07(\mathrm{~s}, 2 \mathrm{H}, \mathrm{ArH})$, 7.20-7.28 (m, 1H, ArH), 7.38-7.44 (m, 1H, ArH), 7.59-7.65 (m, 1H, ArH), 7.88-7.90 (d, 1H, $\mathrm{ArH}, J=9 \mathrm{~Hz}), 8.19(\mathrm{~s}, 1 \mathrm{H},=\mathrm{CH}), 11.97(\mathrm{~s}, 1 \mathrm{H}, \mathrm{NH}) ;{ }^{13} \mathrm{C}$ NMR $\left(150 \mathrm{MHz}, \mathrm{DMSO}-d_{6}\right) \delta$ (ppm): $20.90\left(\mathrm{CH}_{3}\right), 23.20\left(2 \times \mathrm{CH}_{3}\right), 117.62,121.87,126.11,126.72,132.34,133.98,138.23$, $139.92\left(10 \times \mathrm{C}_{\mathrm{ar}}\right), 142.95(=\mathrm{CH}), 155.67,159.19\left(2 \times \mathrm{C}_{\mathrm{ar}}\right)$.

$\mathrm{N}$-[(4-Fluorophenyl)methylidene]-2,4,6-trimethylbenzenesulfonohydrazide (12)

Orange powder; CAS Number: $1799193-67-1$; M.p.: $168{ }^{\circ} \mathrm{C}$; Yield: $40 \%$; ${ }^{1} \mathrm{H}$ NMR (300 MHz, DMSO- $\left.d_{6}\right) \delta(\mathrm{ppm}): 2.23\left(\mathrm{~s}, 3 \mathrm{H}, \mathrm{CH}_{3}\right), 2.63\left(\mathrm{~s}, 6 \mathrm{H}, 2 \times \mathrm{CH}_{3}\right), 7.04(\mathrm{~s}, 2 \mathrm{H}, \mathrm{ArH})$, 7.19-7.25 (m, 2H, ArH), 7.52-7.57 (m, 2H, ArH), $7.90(\mathrm{~s}, 1 \mathrm{H},=\mathrm{CH}), 11.60(\mathrm{~s}, 1 \mathrm{H}, \mathrm{NH}) ;{ }^{13} \mathrm{C}$ NMR $\left(150 \mathrm{MHz}, \mathrm{DMSO}-d_{6}\right) \delta(\mathrm{ppm}): 20.88\left(\mathrm{CH}_{3}\right), 23.19\left(2 \times \mathrm{CH}_{3}\right), 116.29,129.07,132.10$, $133.80,139.67,144.76\left(10 \times \mathrm{C}_{\mathrm{ar}}\right), 142.76(=\mathrm{CH}), 160.89,162.57\left(2 \times \mathrm{C}_{\mathrm{ar}}\right)$.

$N$-[(2-Bromophenyl)methylidene]-2,4,6-trimethylbenzenesulfonohydrazide (13)

Yellow powder; M.p.: $156^{\circ} \mathrm{C}$; Yield: $14 \% ;{ }^{1} \mathrm{H}$ NMR (300 MHz, DMSO- $\left.d_{6}\right) \delta(\mathrm{ppm})$ : $2.24\left(\mathrm{~s}, 3 \mathrm{H}, \mathrm{CH}_{3}\right), 2.63\left(\mathrm{~s}, 6 \mathrm{H}, 2 \times \mathrm{CH}_{3}\right), 7.05(\mathrm{~s}, 2 \mathrm{H}, \mathrm{ArH}), 7.27-7.32(\mathrm{~m}, 1 \mathrm{H}, \mathrm{ArH}), 7.39-7.41$ $(\mathrm{t}, 1 \mathrm{H}, \mathrm{ArH}, J=3 \mathrm{~Hz}), 7.48-7.53(\mathrm{~m}, 1 \mathrm{H}, \mathrm{ArH}), 7.60-7.65(\mathrm{~m}, 1 \mathrm{H}, \mathrm{ArH}), 8.24(\mathrm{~s}, 1 \mathrm{H},=\mathrm{CH})$, $11.88(\mathrm{~s}, 1 \mathrm{H}, \mathrm{NH}) ;{ }^{13} \mathrm{C}$ NMR $\left(150 \mathrm{MHz}, \mathrm{DMSO}-d_{6}\right) \delta(\mathrm{ppm}): 20.90\left(\mathrm{CH}_{3}\right), 23.16\left(2 \times \mathrm{CH}_{3}\right)$, $123.58,126.85,128.61,129.06,132.97,133.68,133.91,139.62\left(10 \times \mathrm{C}_{\mathrm{ar}}\right), 142.93(=\mathrm{CH}), 143.87$, $161.24\left(2 \times \mathrm{C}_{\mathrm{ar}}\right)$.

$\mathrm{N}$-[(3-Bromophenyl)methylidene]-2,4,6-trimethylbenzenesulfonohydrazide (14)

Bright yellow powder; CAS Number: $1799080-75-3$; M.p.: $150{ }^{\circ} \mathrm{C}$; Yield: $73 \% ;{ }^{1} \mathrm{H}$ NMR (300 MHz, DMSO- $\left.d_{6}\right) \delta(\mathrm{ppm}): 2.23\left(\mathrm{~s}, 3 \mathrm{H}, \mathrm{CH}_{3}\right), 2.63\left(\mathrm{~s}, 6 \mathrm{H}, 2 \times \mathrm{CH}_{3}\right), 7.05(\mathrm{~s}, 2 \mathrm{H}$, ArH), 7.31-7.36 (t, 1H, ArH, $J=6 \mathrm{~Hz}, J=9 \mathrm{~Hz}), 7.48-7.57(\mathrm{~m}, 2 \mathrm{H}, \mathrm{ArH}), 7.67-7.68(\mathrm{~m}, 1 \mathrm{H}$, $\mathrm{ArH}), 7.87(\mathrm{~s}, 1 \mathrm{H},=\mathrm{CH}), 11.79(\mathrm{~s}, 1 \mathrm{H}, \mathrm{NH}) ;{ }^{13} \mathrm{C}$ NMR $\left(150 \mathrm{MHz}, \mathrm{DMSO}-d_{6}\right) \delta(\mathrm{ppm}): 20.89$ $\left(\mathrm{CH}_{3}\right), 23.15\left(2 \times \mathrm{CH}_{3}\right), 122.55,125.86,129.35,131.46,132.14,132.84,133.75,136.76,139.69$ $\left(11 \times \mathrm{C}_{\mathrm{ar}}\right), 142.88(=\mathrm{CH}), 143.90\left(\mathrm{C}_{\mathrm{ar}}\right)$.

$\mathrm{N}$-[(4-Bromophenyl)methylidene]-2,4,6-trimethylbenzenesulfonohydrazide (15)

Bright yellow powder; CAS Number: $1799088-79-1$; M.p.: $155^{\circ} \mathrm{C}$; Yield: $67 \% ;{ }^{1} \mathrm{H}$ NMR $\left(300 \mathrm{MHz}, \mathrm{DMSO}-d_{6}\right) \delta(\mathrm{ppm}): 2.24\left(\mathrm{~s}, 3 \mathrm{H}, \mathrm{CH}_{3}\right), 2.62\left(\mathrm{~s}, 6 \mathrm{H}, 2 \times \mathrm{CH}_{3}\right), 7.04(\mathrm{~s}, 2 \mathrm{H}, \mathrm{ArH})$, 7.42-7.45 (d, 2H, ArH, J = 9 Hz), 7.57-7.60 (d, 2H, ArH, J = 9 Hz), $7.88(\mathrm{~s}, 1 \mathrm{H},=\mathrm{CH}), 11.70$ 
(s, $1 \mathrm{H}, \mathrm{NH}) ;{ }^{13} \mathrm{C}$ NMR $\left(150 \mathrm{MHz}, \mathrm{DMSO}-d_{6}\right) \delta(\mathrm{ppm}): 20.89\left(\mathrm{CH}_{3}\right), 23.19\left(2 \times \mathrm{CH}_{3}\right), 123.55$, $128.80,132.12,132.31,133.57,133.76,139.66\left(11 \times \mathrm{C}_{\mathrm{ar}}\right), 142.82(=\mathrm{CH}), 144.53\left(\mathrm{C}_{\mathrm{ar}}\right)$.

$\mathrm{N}$-[(2-Chlorophenyl)methylidene]-2,4,6-trimethylbenzenesulfonohydrazide (16)

Yellow powder; CAS Number: $1799120-48-1$; M.p.: $125^{\circ} \mathrm{C}$; Yield: $35 \% ;{ }^{1} \mathrm{H}$ NMR (300 MHz, DMSO- $\left.d_{6}\right) \delta$ (ppm): $2.24\left(\mathrm{~s}, 3 \mathrm{H}, \mathrm{CH}_{3}\right), 2.63\left(\mathrm{~s}, 6 \mathrm{H}, 2 \times \mathrm{CH}_{3}\right), 7.05(\mathrm{~s}, 2 \mathrm{H}, \mathrm{ArH})$, 7.32-7.41 (m, 1H, ArH), 7.45-7.51 (m, 1H, ArH), 7.57-7.65 (m, 2H, ArH), $8.98(\mathrm{~s}, 1 \mathrm{H},=\mathrm{CH})$, $11.87(\mathrm{~s}, 1 \mathrm{H}, \mathrm{NH}) ;{ }^{13} \mathrm{C}$ NMR $\left(75 \mathrm{MHz}, \mathrm{DMSO}-d_{6}\right) \delta(\mathrm{ppm}): 20.89\left(\mathrm{CH}_{3}\right), 23.17\left(2 \times \mathrm{CH}_{3}\right)$, $126.49,128.68,130.69,131.41,132.17,133.18,135.15,139.64,141.49\left(11 \times \mathrm{C}_{\mathrm{ar}}\right), 142.95(=\mathrm{CH})$, $158.77\left(\mathrm{C}_{\mathrm{ar}}\right)$.

$\mathrm{N}$-[(4-Chlorophenyl)methylidene]-2,4,6-trimethylbenzenesulfonohydrazide (17)

Yellow powder; CAS Number: 1798924-70-5; M.p.: $122{ }^{\circ} \mathrm{C}$; Yield: $29 \% ;{ }^{1} \mathrm{H}$ NMR $(300$ MHz, DMSO- $\left.d_{6}\right) \delta(\mathrm{ppm}): 2.24\left(\mathrm{~s}, 3 \mathrm{H}, \mathrm{CH}_{3}\right), 2.63\left(\mathrm{~s}, 6 \mathrm{H}, 2 \times \mathrm{CH}_{3}\right), 7.04(\mathrm{~s}, 2 \mathrm{H}, \mathrm{ArH}), 7.43-$ $7.46(\mathrm{~d}, 1 \mathrm{H}, \mathrm{ArH}, J=9 \mathrm{~Hz}), 7.49-7.52(\mathrm{~d}, 1 \mathrm{H}, \mathrm{ArH}, J=9 \mathrm{~Hz}), 7.57-7.60(\mathrm{~d}, 1 \mathrm{H}, \mathrm{ArH}, J=9 \mathrm{~Hz})$, 7.89-7.92 (d, 1H, ArH, J = 9 Hz), $8.72(\mathrm{~s}, 1 \mathrm{H},=\mathrm{CH}), 11.70(\mathrm{~s}, 1 \mathrm{H}, \mathrm{NH}) ;{ }^{13} \mathrm{C}$ NMR $(150 \mathrm{MHz}$, DMSO- $\left.d_{6}\right) \delta$ (ppm): $20.89\left(\mathrm{CH}_{3}\right), 23.19\left(2 \times \mathrm{CH}_{3}\right), 128.57,129.29,130.51,132.12,133.09$, $133.23,133.77,134.81,136.56,139.66\left(10 \times \mathrm{C}_{\mathrm{ar}}\right), 142.82(=\mathrm{CH}), 144.44,161.08\left(2 \times \mathrm{C}_{\mathrm{ar}}\right)$.

$\mathrm{N}$-[(3-Chlorophenyl)methylidene]-2,4,6-trimethylbenzenesulfonohydrazide (18)

Bright yellow powder; CAS Number: $1799187-65-7$; M.p.: $140-143{ }^{\circ} \mathrm{C}$; Yield: $41 \% ;{ }^{1} \mathrm{H}$ NMR (600 MHz, DMSO- $\left.d_{6}\right) \delta(\mathrm{ppm}): 2.25\left(\mathrm{~s}, 3 \mathrm{H}, \mathrm{CH}_{3}\right), 2.64\left(\mathrm{~s}, 6 \mathrm{H}, 2 \times \mathrm{CH}_{3}\right), 7.06(\mathrm{~s}, 2 \mathrm{H}$, ArH), 7.40-7.48 (m, 1H, ArH), 7.53-7.54 (m, 1H, ArH), 7.56-7.57 (d, 1H, ArH, J = 6 Hz), 7.60-7.62 (m, 1H, ArH, J = $12 \mathrm{~Hz}), 8.73(\mathrm{~s}, 1 \mathrm{H},=\mathrm{CH}), 11.81(\mathrm{~s}, 1 \mathrm{H}, \mathrm{NH}) ;{ }^{13} \mathrm{C}$ NMR $(150 \mathrm{MHz}$, DMSO- $\left.d_{6}\right) \delta$ (ppm): $20.88\left(\mathrm{CH}_{3}\right), 23.15\left(2 \times \mathrm{CH}_{3}\right), 125.46,126.42,127.39,129.95,132.12$, $134.17,136.25,139.64\left(10 \times \mathrm{C}_{\mathrm{ar}}\right), 142.86(=\mathrm{CH}), 143.98,161.05\left(2 \times \mathrm{C}_{\mathrm{ar}}\right)$.

$\mathrm{N}$-[(2-Chloro-3-methoxyphenyl)methylidene]-2,4,6-trimethylbenzenesulfonohydrazide (19)

Bright yellow powder; M.p.: $158-160{ }^{\circ} \mathrm{C}$; Yield: $47 \%$; ${ }^{1} \mathrm{H}$ NMR $\left(600 \mathrm{MHz}\right.$, DMSO- $\left.d_{6}\right)$ $\delta(\mathrm{ppm}): 2.25\left(\mathrm{~s}, 3 \mathrm{H}, \mathrm{CH}_{3}\right), 2.63\left(\mathrm{~s}, 6 \mathrm{H}, 2 \times \mathrm{CH}_{3}\right), 3.85\left(\mathrm{~s}, 3 \mathrm{H}, \mathrm{OCH}_{3}\right), 7.06(\mathrm{~s}, 2 \mathrm{H}, \mathrm{ArH})$, 7.14-7.16 (m, 1H, ArH), 7.30-7.35 (m, 1H, ArH), 7.74-7.75 (d, 1H, ArH, J = 6 Hz), $8.31(\mathrm{~s}, 1 \mathrm{H}$, $=\mathrm{CH}), 11.87(\mathrm{~s}, 1 \mathrm{H}, \mathrm{NH}) ;{ }^{13} \mathrm{C}$ NMR $\left(150 \mathrm{MHz}, \mathrm{DMSO}-d_{6}\right) \delta(\mathrm{ppm}): 20.89\left(\mathrm{CH}_{3}\right), 23.15(2 \times$ $\left.\mathrm{CH}_{3}\right), 56.93\left(\mathrm{OCH}_{3}\right), 115.77,117.87,119.94,123.52,128.50,132.15,139.63,141.76\left(10 \times \mathrm{C}_{\mathrm{ar}}\right)$, $142.92(=\mathrm{CH}), 155.37,158.99\left(2 \times \mathrm{C}_{\mathrm{ar}}\right)$.

$\mathrm{N}$-[(3-Chloro-4-methoxyphenyl)methylidene]-2,4,6-trimethylbenzenesulfonohydrazide (20)

Yellow powder; M.p.: $159-162{ }^{\circ} \mathrm{C}$; Yield: $3 \% ;{ }^{1} \mathrm{H}$ NMR $\left(600 \mathrm{MHz}\right.$, DMSO- $\left.d_{6}\right) \delta(\mathrm{ppm})$ : $2.26\left(\mathrm{~s}, 3 \mathrm{H}, \mathrm{CH}_{3}\right), 2.62\left(\mathrm{~s}, 6 \mathrm{H}, 2 \times \mathrm{CH}_{3}\right), 3.89\left(\mathrm{~s}, 3 \mathrm{H}, \mathrm{OCH}_{3}\right), 7.05(\mathrm{~s}, 2 \mathrm{H}, \mathrm{ArH}), 7.29-7.30$ $(\mathrm{d}, 1 \mathrm{H}, \mathrm{ArH}, J=6 \mathrm{~Hz}), 7.82-7.84(\mathrm{~d}, 1 \mathrm{H}, \mathrm{ArH}, J=12 \mathrm{~Hz}), 7.94-7.95(\mathrm{~d}, 1 \mathrm{H}, \mathrm{ArH}, J=6 \mathrm{~Hz})$, $8.65(\mathrm{~s}, 1 \mathrm{H},=\mathrm{CH}), 11.88(\mathrm{~s}, 1 \mathrm{H}, \mathrm{NH}) ;{ }^{13} \mathrm{C}$ NMR $\left(150 \mathrm{MHz}, \mathrm{DMSO}-d_{6}\right) \delta(\mathrm{ppm}): 20.95\left(\mathrm{CH}_{3}\right)$, $24.28\left(2 \times \mathrm{CH}_{3}\right), 56.93\left(\mathrm{OCH}_{3}\right), 111.50,113.55,122.14,127.82,129.42,129.65,132.99,133.12$ $\left(10 \times \mathrm{C}_{\mathrm{ar}}\right), 142.15(=\mathrm{CH}), 157.24,160.43\left(2 \times \mathrm{C}_{\mathrm{ar}}\right)$.

$\mathrm{N}$-[(3-Bromo-4-methoxyphenyl)methylidene]-2,4,6-trimethylbenzenesulfonohydrazide (21)

Pink powder; CAS Number: $1799222-06-2$; M.p.: $158-160{ }^{\circ} \mathrm{C}$; Yield: $76 \%$; ${ }^{1} \mathrm{H}$ NMR $\left(600 \mathrm{MHz}, \mathrm{DMSO}-d_{6}\right) \delta(\mathrm{ppm}): 2.25\left(\mathrm{~s}, 3 \mathrm{H}, \mathrm{CH}_{3}\right), 2.64\left(\mathrm{~s}, 6 \mathrm{H}, 2 \times \mathrm{CH}_{3}\right), 3.86\left(\mathrm{~s}, 3 \mathrm{H}, \mathrm{OCH}_{3}\right)$, $7.05(\mathrm{~s}, 2 \mathrm{H}, \mathrm{ArH}), 7.13-7.14(\mathrm{~d}, 1 \mathrm{H}, \mathrm{ArH}, J=6 \mathrm{~Hz}), 7.49-7.50(\mathrm{~m}, 1 \mathrm{H}, \mathrm{ArH}), 7.71-7.72(\mathrm{~d}, 1 \mathrm{H}$, $\mathrm{ArH}, J=6 \mathrm{~Hz}), 7.83(\mathrm{~s}, 1 \mathrm{H},=\mathrm{CH}), 11.56(\mathrm{~s}, 1 \mathrm{H}, \mathrm{NH}) ;{ }^{13} \mathrm{C}$ NMR $\left(150 \mathrm{MHz}, \mathrm{DMSO}-d_{6}\right) \delta$ (ppm): $20.88\left(\mathrm{CH}_{3}\right), 23.18\left(2 \times \mathrm{CH}_{3}\right), 56.90\left(\mathrm{OCH}_{3}\right), 111.50,113.30,127.98,128.39,131.00$, $132.09,133.82,139.63\left(10 \times \mathrm{C}_{\mathrm{ar}}\right), 142.75(=\mathrm{CH}), 144.14,156.96\left(2 \times \mathrm{C}_{\mathrm{ar}}\right)$.

$\mathrm{N}$-[(5-Bromo-2-hydroxyphenyl)methylidene]-2,4,6-trimethylbenzenesulfonohydrazide (22)

Yellow powder; CAS Number: $1799182-63-0 ;$ M.p.: $160-163{ }^{\circ} \mathrm{C}$; Yield: $49 \% ;{ }^{1} \mathrm{H}$ NMR $\left(600 \mathrm{MHz}, \mathrm{DMSO}-d_{6}\right) \delta(\mathrm{ppm}): 2.26\left(\mathrm{~s}, 3 \mathrm{H}, \mathrm{CH}_{3}\right), 2.62\left(\mathrm{~s}, 6 \mathrm{H}, 2 \times \mathrm{CH}_{3}\right), 6.81-6.82(\mathrm{~d}, 1 \mathrm{H}$, $\mathrm{ArH}, J=6 \mathrm{~Hz}), 6.96-6.97(\mathrm{~d}, 1 \mathrm{H}, \mathrm{ArH}, J=6 \mathrm{~Hz}), 7.07(\mathrm{~s}, 2 \mathrm{H}, \mathrm{ArH}), 7.51-7.55(\mathrm{~m}, 1 \mathrm{H}, \mathrm{ArH})$, $8.11(\mathrm{~s}, 1 \mathrm{H},=\mathrm{CH}), 10.39(\mathrm{~s}, 1 \mathrm{H}, \mathrm{OH}), 11.70(\mathrm{~s}, 1 \mathrm{H}, \mathrm{NH}) ;{ }^{13} \mathrm{C} \mathrm{NMR}\left(150 \mathrm{MHz}, \mathrm{DMSO}-d_{6}\right) \delta$ (ppm): $20.89\left(\mathrm{CH}_{3}\right), 23.07\left(2 \times \mathrm{CH}_{3}\right), 111.06,118.96,119.40,128.53,132.18,139.59,141.83$ (9 $\left.\times \mathrm{C}_{\mathrm{ar}}\right), 142.94(=\mathrm{CH}), 155.83,158.13,161.21\left(3 \times \mathrm{C}_{\mathrm{ar}}\right)$.

$\mathrm{N}$-[(3,5-Dichloro-2-hydroxyphenyl)methylidene]-2,4,6-trimetylbenzenesulfonohydrazide (23)

Yellow powder; M.p.: $154-158{ }^{\circ} \mathrm{C}$; Yield: $69 \% ;{ }^{1} \mathrm{H}$ NMR $\left(600 \mathrm{MHz}, \mathrm{DMSO}-d_{6}\right) \delta(\mathrm{ppm})$ : $2.26\left(\mathrm{~s}, 3 \mathrm{H}, \mathrm{CH}_{3}\right), 2.62\left(\mathrm{~s}, 6 \mathrm{H}, 2 \times \mathrm{CH}_{3}\right), 7.10(\mathrm{~s}, 2 \mathrm{H}, \mathrm{ArH}), 7.44-7.45(\mathrm{~d}, 1 \mathrm{H}, \mathrm{ArH}, J=6 \mathrm{~Hz})$, 
7.75-7.76 (d, 1H, ArH, J = $6 \mathrm{~Hz}), 8.15(\mathrm{~s}, 1 \mathrm{H},=\mathrm{CH}), 10.72(\mathrm{~s}, 1 \mathrm{H}, \mathrm{OH}), 12.11(\mathrm{~s}, 1 \mathrm{H}, \mathrm{NH}) ;{ }^{13} \mathrm{C}$ NMR (150 MHz, DMSO- $\left.d_{6}\right) \delta(\mathrm{ppm}): 20.91\left(\mathrm{CH}_{3}\right), 22.99\left(2 \times \mathrm{CH}_{3}\right), 120.78,124.0,126.69$, $130.35,132.31,139.60,143.25\left(9 \times \mathrm{C}_{\mathrm{ar}}\right), 143.75(=\mathrm{CH}), 151.37,153.80,164.02\left(3 \times \mathrm{C}_{\mathrm{ar}}\right)$.

$\mathrm{N}$-[(2-Hydroxy-3,5-diiodophenyl)methylidene]-2,4,6-trimethylbenzenesulfonohydrazide (24)

Yellow powder; M.p.: $280{ }^{\circ} \mathrm{C}$; Yield: $66 \% ;{ }^{1} \mathrm{H}$ NMR $\left(600 \mathrm{MHz}, \mathrm{DMSO}-d_{6}\right) \delta(\mathrm{ppm})$ : $2.27\left(\mathrm{~s}, 3 \mathrm{H}, \mathrm{CH}_{3}\right), 2.61\left(\mathrm{~s}, 6 \mathrm{H}, 2 \times \mathrm{CH}_{3}\right), 7.10(\mathrm{~s}, 2 \mathrm{H}, \mathrm{ArH}), 7.74-7.75(\mathrm{~d}, 1 \mathrm{H}, \mathrm{ArH}, J=6 \mathrm{~Hz})$, 7.99-8.00 (d, 1H, ArH, J = $6 \mathrm{~Hz}), 8.03(\mathrm{~s}, 1 \mathrm{H},=\mathrm{CH}), 11.04(\mathrm{~s}, 1 \mathrm{H}, \mathrm{OH}), 12.20(\mathrm{~s}, 1 \mathrm{H}, \mathrm{NH})$; ${ }^{13} \mathrm{C}$ NMR $\left(150 \mathrm{MHz}\right.$, DMSO- $\left.d_{6}\right) \delta$ (ppm): $20.91\left(\mathrm{CH}_{3}\right), 22.94\left(2 \times \mathrm{CH}_{3}\right), 83.40,88.67,121.45$, $132.37,132.96,138.26,139.58\left(9 \times \mathrm{C}_{\mathrm{ar}}\right), 143.34(=\mathrm{CH}), 145.43,146.98,155.86\left(3 \times \mathrm{C}_{\mathrm{ar}}\right)$.

$\mathrm{N}$-[(2-Bromo-3-hydroxy-4-methoxyphenyl)methylidene]-2,4,6-trimethylbenzenesulfono hydrazide (25)

Brown powder; CAS Number: 1799215-12-5; M.p.: $180{ }^{\circ} \mathrm{C}$; Yield: 55\%; ${ }^{1} \mathrm{H}$ NMR (600 MHz, DMSO- $\left.d_{6}\right) \delta(\mathrm{ppm}): 2.25\left(\mathrm{~s}, 3 \mathrm{H}, \mathrm{CH}_{3}\right), 2.63\left(\mathrm{~s}, 6 \mathrm{H}, 2 \times \mathrm{CH}_{3}\right), 3.83\left(\mathrm{~s}, 3 \mathrm{H}, \mathrm{OCH}_{3}\right)$, 7.02-7.03 (d, 1H, ArH, J = $6 \mathrm{~Hz}), 7.05(\mathrm{~s}, 2 \mathrm{H}, \mathrm{ArH}), 7.11-7.13(\mathrm{~m}, 1 \mathrm{H}, \mathrm{ArH}), 8.20(\mathrm{~s}, 1 \mathrm{H}$, $=\mathrm{CH}), 8.91(\mathrm{~s}, 1 \mathrm{H}, \mathrm{OH}), 11.61(\mathrm{~s}, 1 \mathrm{H}, \mathrm{NH}) ;{ }^{13} \mathrm{C} \mathrm{NMR}\left(150 \mathrm{MHz}, \mathrm{DMSO}-d_{6}\right) \delta(\mathrm{ppm}): 20.89$ $\left(\mathrm{CH}_{3}\right), 23.20\left(2 \times \mathrm{CH}_{3}\right), 56.80\left(\mathrm{OCH}_{3}\right), 111.57,117.25,119.60,125.89,132.10,139.61,142.76$, $144.16\left(10 \times \mathrm{C}_{\mathrm{ar}}\right), 144.92(=\mathrm{CH}), 149.85,161.11\left(2 \times \mathrm{C}_{\mathrm{ar}}\right)$.

2,4,6-Trimethyl- $N$-(phenylmethylidene)benzenesulfonohydrazide (26)

White powder; CAS Number: $16182-18-6$; M.p.: $131-133{ }^{\circ} \mathrm{C}$; Yield: $10 \%$; ${ }^{1} \mathrm{H}$ NMR $\left(600 \mathrm{MHz}, \mathrm{DMSO}-d_{6}\right) \delta(\mathrm{ppm}): 2.24\left(\mathrm{~s}, 3 \mathrm{H}, \mathrm{CH}_{3}\right), 2.65\left(\mathrm{~s}, 6 \mathrm{H}, 2 \times \mathrm{CH}_{3}\right), 7.05(\mathrm{~s}, 2 \mathrm{H}, \mathrm{ArH})$, 7.35-7.40 (m, 3H, ArH), 7.49-7.51 (m, 2H, ArH), $7.91(\mathrm{~s}, 1 \mathrm{H},=\mathrm{CH}), 11.59(\mathrm{~s}, 1 \mathrm{H}, \mathrm{NH}) ;{ }^{13} \mathrm{C}$ NMR (150 MHz, DMSO- $\left.d_{6}\right) \delta(\mathrm{ppm}): 20.88\left(\mathrm{CH}_{3}\right), 23.21\left(2 \times \mathrm{CH}_{3}\right), 126.95,129.29,130.35$, $132.10,133.82,134.30,139.68,142.76\left(12 \times \mathrm{C}_{\mathrm{ar}}\right), 145.73(=\mathrm{CH})$.

Examples of ${ }^{1} \mathrm{H}$ NMR and ${ }^{13} \mathrm{C}$ NMR spectra of synthesized 2,4,6-trimethylbenzenesulfonyl hydrazones are presented in the Supplementary Materials (Figures S1-S8).

\subsection{Microbiology \\ In Vitro Antimicrobial Activity Assay}

The examined compounds $\mathbf{2 - 2 6}$ were screened in vitro for antibacterial and antifungal activities according to the procedure described earlier by our group $[35,36]$ with the use of the protocols of European Committee on Antimicrobial Susceptibility Testing (EUCAST) [37] and Clinical and Laboratory Standards Institute guidelines [38]. All the experiments were repeated three times, and representative data are presented. Detailed procedure for the in vitro antimicrobial activity assay is presented in the Supplementary Materials.

\section{Results and Discussion}

\subsection{Chemistry}

The 2,4,6-trimethylbenzenesulfonyl hydrazones 2-26 were synthesized on the basis of one-step condensation reaction of 2,4,6-timethylbenzenesufonohydrazide (1) with appropriate substituted benzaldehydes (Scheme 1). Among synthesized 2,4,6-trimethylbenzenesulfonyl hydrazones 2-26, nine compounds had not been described earlier in scientific literature. Yield of the synthesis ranged from 3 to $78 \%$. The highest yield was obtained for compound $8-\mathrm{N}$ [(3-iodophenyl)methylidene]-2,4,6-trimethylbenzenesulfonohydrazide, whereas the lowest was for derivative 20-N-[(3-chloro-4-methoxyphenyl)methylidene]-2,4,6-trimethylbenzenesul fonohydrazide. Synthesized compounds are stable solids and can be dissolved in DMSO at ambient temperature. All of synthesized compounds were identified by the analysis of ${ }^{1} \mathrm{H}$ NMR and ${ }^{13} \mathrm{C}$ NMR spectra.

The chemical structure of synthesized 2,4,6-trimethylbenzenesulfonyl hydrazones (2-26) was established with the use of the analysis of ${ }^{1} \mathrm{H}$ NMR and ${ }^{13} \mathrm{C}$ NMR spectra. Compounds 2-26 on the ${ }^{1} \mathrm{H}$ NMR spectra possessed two characteristic singlet signals. First of them in the range of $\delta 7.83-8.98 \mathrm{ppm}$ corresponded to proton in $=\mathrm{CH}$ group and confirmed the successful conduction of condensation reaction, whereas the other at $\delta 11.23-$ $12.20 \mathrm{ppm}$ corresponded to proton in NH group. In ${ }^{13} \mathrm{C}$ NMR spectra for compounds 2-26, 
we found peak for carbon atom of $=\mathrm{CH}$ group at $\delta 142.15-147.57 \mathrm{ppm}$. Other aliphatic and aromatic fragments of synthesized molecules in the ${ }^{1} \mathrm{H}$ and ${ }^{13} \mathrm{C}$ NMR spectra were found at the expected range of chemical shift.

\subsection{Antimicrobial Activity}

The antimicrobial activities of 2,4,6-timethylbenzenesufonohydrazide (1) and synthesized 2,4,6-trimethylbenzenesulfonyl hydrazones 2-26 were tested against reference Grampositive (eight strains) and Gram-negative (six strains) bacteria. Moreover, antifungal effect towards yeasts belonging to Candida spp. was investigated. The results are shown in Table 1; Table 2. All tested 2,4,6-trimethylbenzenesulfonyl hydrazones 2-26 did not have activity against Gram-negative bacteria and fungi. The 2,4,6-timethylbenzenesufonohydrazide (1) showed moderate to mild antibacterial activity against Gram-negative bacterial strains (MIC $=250-1000 \mu \mathrm{g} / \mathrm{mL}$ ) and similar antifungal activity against fungi from Candida spp. with MIC values in the range of 250-1000 $\mu \mathrm{g} / \mathrm{mL}$. Moreover, among 2,4,6-trimethy lbenzenesulfonyl hydrazones, compounds $5,8,10,11,13,14,18$, and 26 were inactive towards all microorganisms from ATCC. The rest of the compounds displayed some antibacterial activity against Gram-positive bacteria. The microorganisms from Staphylococcus spp., Enterococcus faecalis ATCC 29212, Micrococcus luteus ATCC 10240, and Bacillus spp. were especially sensitive to compounds $7, \mathbf{2 2}, \mathbf{2 3}, \mathbf{2 4}$, and $\mathbf{2 5}$. Minimal inhibitory concentration (MIC) of these derivatives, which inhibited growth of bacteria, ranged from 7.81 to 500 $\mu \mathrm{g} / \mathrm{mL}(\mathrm{MBC}=7.81 \rightarrow 1000 \mu \mathrm{g} / \mathrm{mL})$.

Among them, especially compound 24, exhibited a very strong bactericidal effect towards micrococci and bacilli (MIC $=7.81 \mu \mathrm{g} / \mathrm{mL}$ and $\mathrm{MBC}=7.81-15.62 \mu \mathrm{g} / \mathrm{mL}$ ) and strong bactericidal effect against staphylococci and enterococci (MIC $=15.62 \mu \mathrm{g} / \mathrm{mL}$ and $\mathrm{MBC}=15.62-62.5 \mu \mathrm{g} / \mathrm{mL}$ ). Similarly, compound 23 showed very strong or strong activity $(\mathrm{MIC}=7.81-15.62 \mu \mathrm{g} / \mathrm{mL}$ and $\mathrm{MBC}=15.62 \mu \mathrm{g} / \mathrm{mL}$ ) towards M. luteus ATCC 10240 and Bacillus spp., and slightly lower (mainly moderate) against remaining bacteria.

In turn, compound 25 exhibited good activity against Gram-positive bacteria (MIC $=62.5-125 \mu \mathrm{g} / \mathrm{mL}$ and $\mathrm{MBC}=125->1000 \mu \mathrm{g} / \mathrm{mL}$ ), except S. aureus ATCC 43300 and $E$. faecalis ATCC 29212 (moderate effect with MIC $=250 \mu \mathrm{g} / \mathrm{mL}$ and $\mathrm{MBC}=>1000 \mu \mathrm{g} / \mathrm{mL}$ ).

Almost all bacteria (except enterococci) were also sensitive to compound 22. Its minimal concentrations, which inhibited growth of bacteria, ranged from $31.25 \mu \mathrm{g} / \mathrm{mL}$ in the case of B. subtilis ATCC 6633 to $500 \mu \mathrm{g} / \mathrm{mL}$ for S. aureus strains (MBC $=500->1000$ $\mu \mathrm{g} / \mathrm{mL}$ ).

The other compounds showed moderate or mild activity against Gram-positive bacteria with MIC from 250 to $1000 \mu \mathrm{g} / \mathrm{mL}$, or had no activity.

In relation to the antibacterial activity of reference substances, it is worth underlining that the activity of compound $\mathbf{2 4}$ possessed 2-hydroxy-3,5-diiodophenyl substituent against M. luteus ATCC $10240(\mathrm{MIC}=7.81 \mu \mathrm{g} / \mathrm{mL})$, which was eight times greater that the activity of nitrofurantoin (MIC $=62.5 \mu \mathrm{g} / \mathrm{mL}$ ). Towards B. subtilis ATCC 6633, this compound showed two times higher $(\mathrm{MIC}=7.81 \mu \mathrm{g} / \mathrm{mL}$ ) activity than cefuroxime $(\mathrm{MIC}=15.62 \mu \mathrm{g} / \mathrm{mL}$ ) and eight times higher activity than ampicillin (MIC $=62.5 \mu \mathrm{g} / \mathrm{mL}$ ). Activity of this hydrazone towards B. cereus ATCC 10876 was equal to the activity of nitrofurantoin (MIC $=7.81$ $\mu \mathrm{g} / \mathrm{mL}$ ) and four times greater than the activity of cefuroxime $(\mathrm{MIC}=31.25 \mu \mathrm{g} / \mathrm{mL}$ ). Towards Staphylococcus aureus ATCC 29213 and ATCC 25923, its activity was equal to the activity of nitrofurantoin (MIC $=15.62 \mu \mathrm{g} / \mathrm{mL}$ ). 


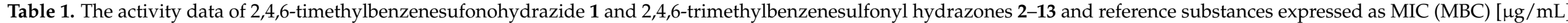
and $\{\mathrm{MBC} / \mathrm{MIC}\}$ ratio against the reference strains of Gram-positive bacteria.

\begin{tabular}{|c|c|c|c|c|c|c|c|c|c|c|c|c|c|}
\hline \multirow{2}{*}{\multicolumn{2}{|c|}{ Species }} & \multicolumn{12}{|c|}{ MIC (MBC) $[\mu \mathrm{g} / \mathrm{mL}]$ and $\{\mathrm{MBC} / \mathrm{MIC}\}$ Ratio of the Studied Compounds and Positive Controls } \\
\hline & & 1 & 2 & 3 & 4 & 6 & 7 & 9 & 12 & CIP/VA* & NIT & CFX & APC \\
\hline \multirow{8}{*}{$\begin{array}{l}\text { Gram- } \\
\text { positive } \\
\text { bacteria }\end{array}$} & $\begin{array}{c}\text { Staphylococcus } \\
\text { aureus } \\
\text { ATCC } 25923\end{array}$ & $\begin{array}{c}250 \\
(500) \\
\{2\}\end{array}$ & - & $\begin{array}{c}500 \\
(>1000) \\
\{>2\}\end{array}$ & $\begin{array}{c}1000 \\
(>1000) \\
\{>1\}\end{array}$ & $\begin{array}{c}1000 \\
(>1000) \\
\{>1\}\end{array}$ & $\begin{array}{c}1000 \\
(>1000) \\
\{>1\}\end{array}$ & $\begin{array}{c}1000 \\
(>1000) \\
\{>1\}\end{array}$ & - & $\begin{array}{c}0.48 \\
(0.48) \\
\{1\} \\
\end{array}$ & $\begin{array}{c}15.62 \\
(15.62)\end{array}$ & 0.49 & nd \\
\hline & $\begin{array}{c}\text { Staphylococcus } \\
\text { aureus } \\
\text { ATCC } 43300\end{array}$ & $\begin{array}{c}500 \\
(500) \\
\{1\}\end{array}$ & - & $\begin{array}{c}1000 \\
(>1000) \\
\{>1\}\end{array}$ & - & - & $\begin{array}{c}1000 \\
(>1000) \\
\{>1\}\end{array}$ & $\begin{array}{c}1000 \\
(>1000) \\
\{>1\}\end{array}$ & - & $\begin{array}{c}0.24 \\
(0.24) \\
\{1\} \\
\end{array}$ & $\begin{array}{c}7.81 \\
(15.62)\end{array}$ & nd & nd \\
\hline & $\begin{array}{c}\text { Staphylococcus } \\
\text { aureus } \\
\text { ATCC } 29213\end{array}$ & $\begin{array}{c}500 \\
(500) \\
\{1\}\end{array}$ & - & - & - & - & $\begin{array}{c}1000 \\
(>1000) \\
\{>1\}\end{array}$ & $\begin{array}{c}1000 \\
(>1000) \\
\{>1\}\end{array}$ & - & $\begin{array}{c}0.48 \\
(0.48) \\
\{1\} \\
\end{array}$ & $\begin{array}{c}15.62 \\
(15.62)\end{array}$ & nd & nd \\
\hline & $\begin{array}{l}\text { Staphylococcus } \\
\text { epidermidis } \\
\text { ATCC } 12228\end{array}$ & $\begin{array}{c}500 \\
(500) \\
\{1\}\end{array}$ & - & $\begin{array}{c}500 \\
(>1000) \\
\{>2\}\end{array}$ & $\begin{array}{c}500 \\
(>1000) \\
\{>2\}\end{array}$ & $\begin{array}{c}1000 \\
(>1000) \\
\{>1\}\end{array}$ & $\begin{array}{c}1000 \\
(>1000) \\
\{>1\}\end{array}$ & $\begin{array}{c}1000 \\
(>1000) \\
\{>1\}\end{array}$ & - & $\begin{array}{c}0.12 \\
(0.12) \\
\{1\} \\
\end{array}$ & $\begin{array}{c}3.91 \\
(7.81)\end{array}$ & 0.24 & nd \\
\hline & $\begin{array}{l}\text { Enterococcus } \\
\text { faecalis } \\
\text { ATCC } 29212\end{array}$ & $\begin{array}{c}1000 \\
(>1000) \\
\{>1\}\end{array}$ & - & $\begin{array}{c}500 \\
(>1000) \\
\{>2\}\end{array}$ & - & - & - & - & - & $\begin{array}{c}0.98^{*} \\
(1.95) \\
\{2\}\end{array}$ & $\begin{array}{c}7.81 \\
(7.81)\end{array}$ & nd & nd \\
\hline & $\begin{array}{c}\text { Micrococcus } \\
\text { luteus } \\
\text { ATCC } 10240\end{array}$ & $\begin{array}{c}500 \\
(500) \\
\{1\}\end{array}$ & - & - & - & $\begin{array}{c}1000 \\
(>1000) \\
\{>1\}\end{array}$ & $\begin{array}{c}250 \\
(>1000) \\
\{>4\}\end{array}$ & - & $\begin{array}{c}250 \\
(>1000) \\
\{>4\}\end{array}$ & $\begin{array}{c}0.98 \\
(1.95) \\
\{2\} \\
\end{array}$ & $\begin{array}{l}62.5 \\
(62.5)\end{array}$ & 0.98 & nd \\
\hline & $\begin{array}{c}\text { Bacillus } \\
\text { subtilis } \\
\text { ATCC } 6633\end{array}$ & $\begin{array}{c}500 \\
(500) \\
\{1\}\end{array}$ & $\begin{array}{c}500 \\
(>1000) \\
\{>2\}\end{array}$ & $\begin{array}{c}500 \\
(>1000) \\
\{>2\}\end{array}$ & $\begin{array}{c}250 \\
(1000) \\
\{>4\}\end{array}$ & $\begin{array}{c}125 \\
(>1000) \\
\{>8\}\end{array}$ & $\begin{array}{c}62.5 \\
(>1000) \\
\{>16\}\end{array}$ & $\begin{array}{c}250 \\
(>1000) \\
\{>4\}\end{array}$ & $\begin{array}{c}250 \\
(1000) \\
\{4\} \\
\end{array}$ & $\begin{array}{c}0.03 \\
(0.03) \\
\{1\}\end{array}$ & $\begin{array}{c}3.91 \\
(3.91)\end{array}$ & 15.62 & 62.5 \\
\hline & $\begin{array}{c}\text { Bacillus } \\
\text { cereus } \\
\text { ATCC } 10876\end{array}$ & $\begin{array}{c}500 \\
(1000) \\
\{2\}\end{array}$ & $\begin{array}{c}250 \\
(>1000) \\
\{>4\}\end{array}$ & $\begin{array}{c}1000 \\
(>1000) \\
\{>1\}\end{array}$ & - & $\begin{array}{c}1000 \\
(>1000) \\
\{>1\}\end{array}$ & - & - & - & $\begin{array}{c}0.06 \\
(0.12) \\
\{2\}\end{array}$ & $\begin{array}{c}7.81 \\
(15.62)\end{array}$ & 31.25 & nd \\
\hline
\end{tabular}

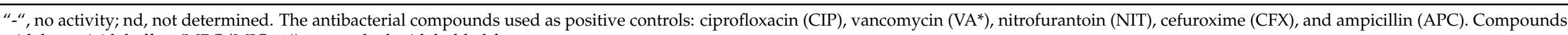
with bactericidal effect (MBC/MIC $\leq 4)$ are marked with bolded font. 


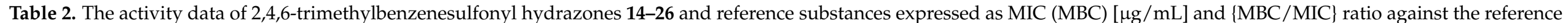
strains of Gram-positive bacteria.

\begin{tabular}{|c|c|c|c|c|c|c|c|c|c|c|c|c|c|c|c|}
\hline \multirow{2}{*}{\multicolumn{2}{|c|}{ Species }} & \multicolumn{14}{|c|}{ MIC (MBC) $[\mu \mathrm{g} / \mathrm{mL}]$ and $\{\mathrm{MBC} / \mathrm{MIC}\}$ Ratio of the Studied Compounds and Positive Controls } \\
\hline & & 15 & 16 & 17 & 19 & 20 & 21 & 22 & 23 & 24 & 25 & CIP/VA* & NIT & CFX & APC \\
\hline \multirow{8}{*}{$\begin{array}{l}\text { Gram- } \\
\text { positive } \\
\text { bacteria }\end{array}$} & $\begin{array}{l}\text { Staphylococcus } \\
\text { aureus } \\
\text { ATCC } \\
25923 \\
\end{array}$ & - & - & - & - & - & - & $\begin{array}{c}500 \\
(>1000) \\
\{>2\}\end{array}$ & $\begin{array}{c}500 \\
(>1000) \\
\{>2\}\end{array}$ & $\begin{array}{c}15.62 \\
(15.62) \\
\{1\}\end{array}$ & $\begin{array}{c}125 \\
(>1000) \\
\{>8\}\end{array}$ & $\begin{array}{c}0.48 \\
(0.48) \\
\{1\}\end{array}$ & $\begin{array}{c}15.62 \\
(15.62)\end{array}$ & 0.49 & nd \\
\hline & $\begin{array}{l}\text { Staphylococcus } \\
\text { aureus } \\
\text { ATCC } \\
43300\end{array}$ & - & - & - & - & - & - & $\begin{array}{c}500 \\
(>1000) \\
\{>2\}\end{array}$ & $\begin{array}{c}500 \\
(>1000) \\
\{>2\}\end{array}$ & $\begin{array}{c}15.62 \\
(15.62) \\
\{1\}\end{array}$ & $\begin{array}{c}250 \\
(>1000) \\
\{>4\}\end{array}$ & $\begin{array}{c}0.24 \\
(0.24) \\
\{1\}\end{array}$ & $\begin{array}{c}7.81 \\
(15.62)\end{array}$ & nd & nd \\
\hline & $\begin{array}{l}\text { Staphylococcus } \\
\text { aureus } \\
\text { ATCC } \\
29213 \\
\end{array}$ & - & $\begin{array}{c}500 \\
(>1000) \\
\{>2\}\end{array}$ & - & - & - & - & $\begin{array}{c}500 \\
(>1000) \\
\{>2\}\end{array}$ & $\begin{array}{c}250 \\
(>1000) \\
\{>4\}\end{array}$ & $\begin{array}{l}15.62 \\
(62.5) \\
\{4\}\end{array}$ & $\begin{array}{c}125 \\
(>1000) \\
\{>8\}\end{array}$ & $\begin{array}{c}0.48 \\
(0.48) \\
\{1\}\end{array}$ & $\begin{array}{c}15.62 \\
(15.62)\end{array}$ & nd & nd \\
\hline & $\begin{array}{l}\text { Staphylococcus } \\
\text { epidermidis } \\
\text { ATCC } \\
12228\end{array}$ & - & $\begin{array}{c}250 \\
(>1000) \\
\{>4\}\end{array}$ & $\begin{array}{c}1000 \\
(>1000) \\
\{>1\}\end{array}$ & $\begin{array}{c}500 \\
(>1000) \\
\{>2\}\end{array}$ & - & - & $\begin{array}{c}125 \\
(500) \\
\{4\}\end{array}$ & $\begin{array}{c}250 \\
(>1000) \\
\{>4\}\end{array}$ & $\begin{array}{c}15.62 \\
(15.62) \\
\{1\}\end{array}$ & $\begin{array}{c}125 \\
(1000) \\
\{8\}\end{array}$ & $\begin{array}{c}0.12 \\
(0.12) \\
\{1\}\end{array}$ & $\begin{array}{c}3.91 \\
(7.81)\end{array}$ & 0.24 & nd \\
\hline & $\begin{array}{c}\text { Enterococcus } \\
\text { faecalis } \\
\text { ATCC } \\
29212 \\
\end{array}$ & - & - & - & - & - & - & - & $\begin{array}{c}125 \\
(>1000) \\
\{>8\}\end{array}$ & $\begin{array}{c}15.62 \\
(62.5) \\
\{4\}\end{array}$ & $\begin{array}{c}250 \\
(>1000) \\
\{>4\}\end{array}$ & $\begin{array}{c}0.98^{*} \\
(1.95) \\
\{2\}\end{array}$ & $\begin{array}{l}7.81 \\
(7.81)\end{array}$ & nd & nd \\
\hline & $\begin{array}{c}\text { Micrococcus } \\
\text { luteus } \\
\text { ATCC } \\
10240\end{array}$ & - & $\begin{array}{c}1000 \\
(>1000) \\
\{>1\}\end{array}$ & $\begin{array}{c}500 \\
(>1000) \\
\{>2\}\end{array}$ & $\begin{array}{c}1000 \\
(>1000) \\
\{>1\}\end{array}$ & $\begin{array}{c}1000 \\
(>1000) \\
\{>1\}\end{array}$ & $\begin{array}{c}1000 \\
(>1000) \\
\{>1\}\end{array}$ & $\begin{array}{c}1000 \\
(>1000) \\
\{>1\}\end{array}$ & $\begin{array}{c}7.81 \\
(15.62) \\
\{2\}\end{array}$ & $\begin{array}{c}7.81 \\
(15.62) \\
\{2\}\end{array}$ & $\begin{array}{c}125 \\
(250) \\
\{2\}\end{array}$ & $\begin{array}{c}0.98 \\
(1.95) \\
\{2\}\end{array}$ & $\begin{array}{l}62.5 \\
(62.5)\end{array}$ & 0.98 & nd \\
\hline & $\begin{array}{c}\text { Bacillus } \\
\text { subtilis } \\
\text { ATCC } 6633\end{array}$ & $\begin{array}{c}1000 \\
(>1000) \\
\{>1\}\end{array}$ & $\begin{array}{c}125 \\
(1000) \\
\{8\}\end{array}$ & $\begin{array}{c}250 \\
(1000) \\
\{4\}\end{array}$ & $\begin{array}{c}250 \\
(1000) \\
\{4\}\end{array}$ & $\begin{array}{c}1000 \\
(>1000) \\
\{>1\}\end{array}$ & $\begin{array}{c}500 \\
(>1000) \\
\{>2\}\end{array}$ & $\begin{array}{l}31.25 \\
(500) \\
\{16\}\end{array}$ & $\begin{array}{c}7.81 \\
(15.62) \\
\{2\}\end{array}$ & $\begin{array}{c}7.81 \\
(7.81) \\
\{1\} \\
\end{array}$ & $\begin{array}{c}62.5 \\
(125) \\
\{2\}\end{array}$ & $\begin{array}{c}0.03 \\
(0.03) \\
\{1\}\end{array}$ & $\begin{array}{c}3.91 \\
(3.91)\end{array}$ & 15.62 & 62.5 \\
\hline & $\begin{array}{l}\text { Bacillus } \\
\text { cereus } \\
\text { ATCC } \\
10876\end{array}$ & $\begin{array}{c}1000 \\
(>1000) \\
\{>1\}\end{array}$ & $\begin{array}{c}1000 \\
(>1000) \\
\{>1\}\end{array}$ & - & - & - & $\begin{array}{c}500 \\
(>1000) \\
\{>2\}\end{array}$ & $\begin{array}{c}250 \\
(1000) \\
\{4\}\end{array}$ & $\begin{array}{c}15.62 \\
(15.62) \\
\{1\}\end{array}$ & $\begin{array}{c}7.81 \\
(15.62) \\
\{2\}\end{array}$ & $\begin{array}{c}125 \\
(>1000) \\
\{>8\}\end{array}$ & $\begin{array}{c}0.06 \\
(0.12) \\
\{2\}\end{array}$ & $\begin{array}{c}7.81 \\
(15.62)\end{array}$ & 31.25 & nd \\
\hline
\end{tabular}

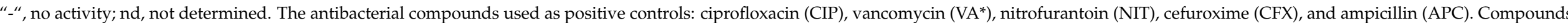
with bactericidal effect (MBC/MIC $\leq 4)$ are marked with bolded font. 


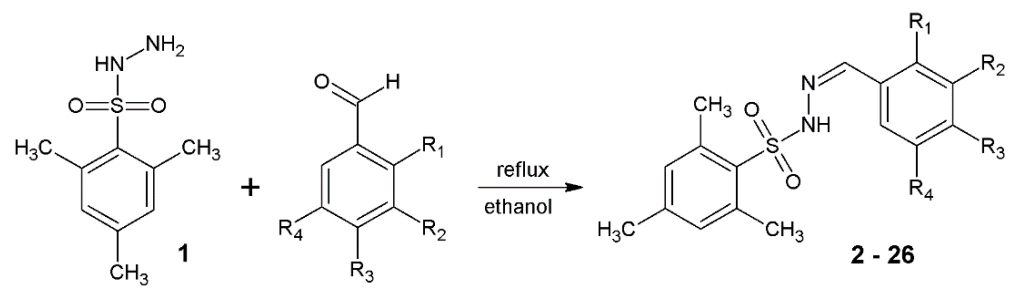

\begin{tabular}{|c|c|c|c|c|}
\hline Compound No & $\mathbf{R}_{1}$ & $\mathbf{R}_{2}$ & $\mathbf{R}_{3}$ & $\mathbf{R}_{4}$ \\
\hline 2 & $\mathrm{OH}$ & $\mathrm{OC}_{2} \mathrm{H}_{5}$ & $\mathrm{H}$ & $\mathrm{H}$ \\
\hline 3 & $\mathrm{H}$ & $\mathrm{OCH}_{3}$ & $\mathrm{OCH}_{3}$ & $\mathrm{H}$ \\
\hline 4 & $\mathrm{H}$ & $\mathrm{OC}_{2} \mathrm{H}_{5}$ & $\mathrm{OH}$ & $\mathrm{H}$ \\
\hline 5 & $\mathrm{OCH}_{3}$ & $\mathrm{H}$ & $\mathrm{OCH}_{3}$ & $\mathrm{H}$ \\
\hline 6 & $\mathrm{OCH}_{3}$ & $\mathrm{OCH}_{3}$ & $\mathrm{H}$ & $\mathrm{H}$ \\
\hline 7 & I & $\mathrm{H}$ & $\mathrm{H}$ & $\mathrm{H}$ \\
\hline 8 & $\mathrm{H}$ & I & $\mathrm{H}$ & $\mathrm{H}$ \\
\hline 9 & $\mathrm{H}$ & $\mathrm{H}$ & $\mathrm{I}$ & $\mathrm{H}$ \\
\hline 10 & $\mathrm{~F}$ & $\mathrm{H}$ & $\mathrm{H}$ & $\mathrm{H}$ \\
\hline 11 & $\mathrm{H}$ & $\mathrm{F}$ & $\mathrm{H}$ & $\mathrm{H}$ \\
\hline 12 & $\mathrm{H}$ & $\mathrm{H}$ & $\mathrm{F}$ & $\mathrm{H}$ \\
\hline 13 & $\mathrm{Br}$ & $\mathrm{H}$ & $\mathrm{H}$ & $\mathrm{H}$ \\
\hline 14 & $\mathrm{H}$ & $\mathrm{Br}$ & $\mathrm{H}$ & $\mathrm{H}$ \\
\hline 15 & $\mathrm{H}$ & $\mathrm{H}$ & $\mathrm{Br}$ & $\mathrm{H}$ \\
\hline 16 & $\mathrm{Cl}$ & $\mathrm{H}$ & $\mathrm{H}$ & $\mathrm{H}$ \\
\hline 17 & $\mathrm{H}$ & $\mathrm{H}$ & $\mathrm{Cl}$ & $\mathrm{H}$ \\
\hline 18 & $\mathrm{H}$ & $\mathrm{Cl}$ & $\mathrm{H}$ & $\mathrm{H}$ \\
\hline 19 & $\mathrm{Cl}$ & $\mathrm{OCH}_{3}$ & $\mathrm{H}$ & $\mathrm{H}$ \\
\hline 20 & $\mathrm{H}$ & $\mathrm{Cl}$ & $\mathrm{OCH}_{3}$ & $\mathrm{H}$ \\
\hline 21 & $\mathrm{H}$ & $\mathrm{Br}$ & $\mathrm{OCH}_{3}$ & $\mathrm{H}$ \\
\hline 22 & $\mathrm{OH}$ & $\mathrm{H}$ & $\mathrm{H}$ & $\mathrm{Br}$ \\
\hline 23 & $\mathrm{OH}$ & $\mathrm{Cl}$ & $\mathrm{H}$ & $\mathrm{Cl}$ \\
\hline 24 & $\mathrm{OH}$ & I & $\mathrm{H}$ & I \\
\hline 25 & $\mathrm{Br}$ & $\mathrm{OH}$ & $\mathrm{OCH}_{3}$ & $\mathrm{H}$ \\
\hline 26 & $\mathrm{H}$ & $\mathrm{H}$ & $\mathrm{H}$ & $\mathrm{H}$ \\
\hline
\end{tabular}

Scheme 1. The synthesis of novel 2,4,6-trimethylbenzenesulfonyl hydrazones.

The highest activity of molecule 23, which was substituted with 2-hydroxy-3,5dichlorophenyl substituent, was shown towards M. luteus ATCC 10240 (MIC $=7.81 \mu \mathrm{g} / \mathrm{mL}$ ), and it was eight times higher than the activity of nitrofurantoin (MIC $=62.5 \mu \mathrm{g} / \mathrm{mL}$ ) against this microorganism, whereas the MIC values of 23 against $B$. subtilis ATCC 6633 (MIC = 7.81) was two times lower than the MIC value of cefuroxime (MIC $=15.62 \mu \mathrm{g} / \mathrm{mL}$ ) and eight times lower than for ampicillin (MIC $=62.5 \mu \mathrm{g} / \mathrm{mL}$ ). It also showed two times higher activity (MIC $=15.62 \mu \mathrm{g} / \mathrm{mL}$ ) towards B. cereus ATCC 10876 than cefuroxime (MIC $=31.25$ $\mu \mathrm{g} / \mathrm{mL}$ ). Additionally, it is worth mentioning that this compound showed bactericidal effect against these microorganisms. In addition to this, the activity of compound 25 substituted with 2-bromo-3-hydroxy-4-methoxyphenyl substituent towards B. subtilis ATCC 6633 was equal to the activity of ampicillin (MIC $=62.5 \mu \mathrm{g} / \mathrm{mL}$ ). The activity data of the most effective compounds $(\mathbf{2 3}, \mathbf{2 4}, \mathbf{2 5})$ in comparison with nitrofurantoin against the reference Gram-positive bacterial strains is presented in graphical way in the Supplementary Materials (Figures S9 and S10).

The highest activity of compound 22, which was substituted with 5-bromo-2-hydroxy phenyl, was displayed against B. subtilis ATCC $6633(\mathrm{MIC}=31.25 \mu \mathrm{g} / \mathrm{mL})$, and it was two times higher than the activity of reference compound ampicillin against this bacterium (MIC $=62.5 \mu \mathrm{g} / \mathrm{mL}$ ). On the other hand, antibacterial activity of compound 7 that possessed 2iodophenyl substituent against B. subtilis ATCC 6633 was equal to the activity of ampicillin (MIC $=62.5 \mu \mathrm{g} / \mathrm{mL}$ ).

Analysis of all obtained results of antimicrobial activity screening also indicates that the conversion of 2,4,6-trimethylbenzenesulfonohydrazide 1 to 2,4,6-trimethylbenzenesulf 
onyl hydrazones resulted in a decrease in activity in some cases. 2,4,6-Trimethylbenzenesulf onyl hydrazone $\mathbf{2 6}$ formed in the reaction of 2,4,6-trimethylbenzenesulfonohydrazide 1 with benzaldehyde was inactive towards all tested bacterial strains. However, the use of aldehydes that contained in the phenyl ring a strong electron-donating substituent, a hydroxy group, and a second electron-withdrawing halogen atom (23-25) increased the activity against Gram-positive bacteria. When analyzing the activity of hydrazide $\mathbf{1}$ and benzenesulfonyl hydrazones, we saw that the free amino group promoted the activity against Staphylococcus aureus ATCC 25923, but the introduction of two halogens into the hydrazone's phenyl ring caused an up to 16-fold increase in activity against this bacterial strain in the case of 2,4,6-trimethylbenzenesulfonyl hydrazone (24). In addition to this, the introduction of two electron-donating substituents in the phenyl ring caused a twofold increase in the activity of the compound 25 against this bacterial strain. The Bacillus subtilis ATCC 6633 was the most sensitive bacterial strain to tested 2,4,6-trimethylbenzenesylfonyl hydrazones. In its case, the free amino group was not conducive to activity.

\section{Conclusions}

In this research we designed, synthesized, and analyzed for potential antimicrobial activity a series of 2,4,6-trimethylbenzenesulfonyl hydrazones 2-26. Our antimicrobial activity assay results indicated that some of the newly obtained compounds 2-26 showed particular activity against Gram-positive bacteria. The highest antibacterial effect was indicated for compounds 7, 22, 23, 24, and 25. The bacteria from Staphylococcus spp., Enterococcus faecalis ATCC 29212, Micrococcus luteus ATCC 10240, and Bacillus spp. were especially sensitive to compound 24 . The minimal inhibitory concentration (MIC) values that inhibited growth of reference microorganisms for this hydrazone ranged from 7.81 to $15.62 \mu \mathrm{g} / \mathrm{mL}$, indicating strong or very strong bactericidal effect of this molecule and its potential application as an antimicrobial agent. The antibacterial activity of the obtained compounds was connected both with the presence of sulfonyl hydrazone moiety in their molecules as well as with substitution with hydroxy and methoxy groups or chlorine and iodine atoms in the phenyl ring. The more substituents that were present in the aromatic ring of an aldehyde, which was used for the condensation reaction, the higher the observed activity of the resulting 2,4,6-trimethylbenzenesulfonyl hydrazones. The most potent compounds against tested bacterial strains will be applied as ligands for the synthesis of metal complexes.

Supplementary Materials: The following are available online at https:/ / www.mdpi.com/article/10.339 0/ma14112723/s1. Figure S1. ${ }^{1} \mathrm{H}$ NMR spectrum of compound 7- $\mathrm{N}$-[(2-iodophenyl)methylidene]-2,4,6trimethylbenzenesulfonohydrazide. Figure S2. ${ }^{13} \mathrm{C}$ NMR spectrum of compound 7-N-[(2-iodophenyl) methylidene]-2,4,6-trimethylbenzenesulfonohydrazide. Figure S3. ${ }^{1} \mathrm{H}$ NMR spectrum of compound 15- $N$-[(4-bromophenyl)methylidene]-2,4,6-trimethylbenzenesulfonohydrazide. Figure S4. ${ }^{13} \mathrm{C}$ NMR spectrum of compound 15- N-[(4-bromophenyl)methylidene]-2,4,6-trimethylbenzenesulfonohydrazide. Figure S5. ${ }^{1} \mathrm{H}$ NMR spectrum of compound 21-N-[(3-bromo-4-methoxyphenyl)methylidene]-2,4,6trimethylbenzenesulfonohydrazide. Figure S6. ${ }^{13} \mathrm{C}$ NMR spectrum of compound 21-N-[(3-bromo-4methoxyphenyl)methylidene]-2,4,6-trimethylbenzenesulfonohydrazide. Figure S7. ${ }^{1} \mathrm{H}$ NMR spectrum of compound 26-2,4,6-trimethyl- $N$-(phenylmethylidene)benzenesulfonohydrazide. Figure $S 8 .{ }^{13} \mathrm{C}$ NMR spectrum of compound 26-2,4,6-trimethyl- $N$-(phenylmethylidene)benzenesulfonohydrazide. Figure $\mathrm{S} 9$. The activity data of the most active compounds: (a) 23, (b) 24, (c) 25, and (d) nitrofurantoin against the reference Gram-positive bacterial strains used in antimicrobial activity study. Figure S10. The activity data of the most active compounds $(23,24$, and 24$)$ compared to nitrofurantoin and expressed as MIC $(\mu \mathrm{g} / \mathrm{mL})$ against the reference Gram-positive bacterial strains.

Author Contributions: Ł.P. designed the study, participated in the synthesis of new 2,4,6-trimethylben zenesulfonyl hydrazones, performed the analysis of the spectral data of obtained compounds, wrote the first draft of the manuscript with the exception of the antimicrobial activity section, and critically revised the final version of the manuscript. S.S. participated in the synthesis of new 2,4,6-trimethylben zenesulfonyl hydrazones. A.B. performed the antimicrobial activity analysis of the obtained compounds and wrote the associated section of the manuscript. M.W. participated in the discussion 
section of the manuscript and critically revised the final version of manuscript. All authors have read and agreed to the published version of the manuscript.

Funding: This research received no external funding.

Institutional Review Board Statement: Not applicable.

Informed Consent Statement: Not applicable.

Data Availability Statement: Data are contained within the article or supplementary material.

Conflicts of Interest: The authors declare no conflict of interest.

\section{References}

1. Moellering, R.C., Jr. Discovering new antimicrobial agents. Inter. J. Antimicrob. Agents 2011, 37, 2-9. [CrossRef]

2. Prestinaci, F.; Pezzotti, P.; Pantosti, A. Antimicrobial resistance: A global multifaceted phenomenon. Pathog. Glob. Health 2015, 109, 309-318. [CrossRef]

3. Morehead, M.S.; Scarbrough, C. Emergence of Global Antibiotic Resistance. Prim. Care Clin. Off. Pract. 2018, 45, 467-484. [CrossRef]

4. Barluenga, J.; Moriel, P.; Valdés, C.; Aznar, F. N-Tosylohydrazones as Reagent for Cross-Coupling Reactions: A Route to Polysubtituted Olefins. Angew. Chem. Int. Ed. 2007, 46, 5587-5590. [CrossRef]

5. Cunha, M.R.; Tavares, M.T.; Carvalho, C.F.; Silva, N.A.T.; Souza, A.D.F.; Pereira, G.J.V.; Ferreira, F.F.; Parise-Filho, R. Environmentaly Safe Condition for the Synthesis of Aryl and Alkyl Sulfonyl Hydrazones via One-Pot Reaction. ACS Sustain. Chem. Eng. 2016, 4, 1899-1905. [CrossRef]

6. Yang, F.-L.; Tian, S.-K. Sulfonyl hydrazides as sulfonyl sources in organic synthesis. Tetrahedron Lett. 2017, 58, 487-504. [CrossRef]

7. Arunprasath, D.; Bala, B.D.; Sekar, G. Luxury of N-Tosylhydrazones in Transition-Metal-Free Transformations. Adv. Synth. Catal. 2018, 361, 1172-1207. [CrossRef]

8. Zhang, D.; Ma, Y.; Liu, Y.; Liu, Z.P. Synthesis of Sulfonylhydrazone- and Acylhydrazone-Substituted 8-Ethoxy-3-nitro-2Hchromones as Potent Antiproliferative and Apoptosis Inducing Agents. Arch. Pharm. 2014, 347, 576-588. [CrossRef]

9. Xie, Z.; Song, Y.; Xu, L.; Guo, Y.; Zhang, M.; Li, L.; Chen, K.; Liu, X. Rapid Synthesis of N-Tosylhydrazones under Solvent-Free Conditions and Their Potential Application Against Human Triple-Negative Breast Cancer. Chem. Open 2018, 7, 977-983. [CrossRef]

10. Wei, D.-C.; Pan, Y.; Wang, H.; Xu, W.-J.; Chen, C.; Zheng, J.-H.; Cai, D. Synthesis of substituted aromatic heterocyclic sulfonyl hydrazone compounds and in vitro anti-hepatoma activity: Preliminary results. Eur. Rev. Med. Pharm. Sci. 2018, 22, $4720-4729$.

11. Popiołek, Ł.; Gawrońska-Grzywacz, M.; Berecka-Rycerz, A.; Paruch, K.; Piątkowska-Chmiel, I.; Natorska-Chomicka, D.; Herbet, M.; Gumieniczek, A.; Dudka, J.; Wujec, M. New benzenesulphonohydrazide derivatives as potential antitumour agents. Oncol. Lett. 2020, 20. [CrossRef] [PubMed]

12. Yang, K.; Yang, J.-Q.; Luo, S.-H.; Mei, W.-J.; Lin, J.-Y.; Zhan, J.-Q.; Wang, Z.-Y. Synthesis of N-2(5H)-furanonyl sulfonyl hydrazone derivatives and their biological evaluation in vitro and in vivo activity against MCF-7 breast cancer cells. Bioorg. Chem. 2021, 107, 104518. [CrossRef] [PubMed]

13. Siemann, S.; Evanoff, D.P.; Marrone, L.; Clarke, A.J.; Viswanatha, T.; Dmitrienko, G. N-Arylsulfonyl Hydrazones as Inhibitors of IMP-1 Metallo- $\beta$-Lactamase. Antimicrob. Agent Chemother. 2002, 46, 2450-2457. [CrossRef] [PubMed]

14. Ghiya, S.; Joshi, Y.C. Synthesis and antimicrobial evaluation of hydrazones derived from 4-methylbenzenesulfonohydrazide in aqueous medium. Med. Chem. Res. 2016, 25, 970-976. [CrossRef]

15. Bhat, M.; Poojary, B.; Kumar, S.M.; Hussain, M.M.; Pai, N.; Revanasiddappa, B.C.; Byrappa, K. Structural, crystallographic, Hirshfeld surface, thermal and antimicrobial evaluation of new sulfonyl hydrazones. J. Mol. Struct. 2018, 1159, 55-66. [CrossRef]

16. Zhou, Z.; Wang, X.; Zhou, T. Synthesis and Antibacterial Activity of Benzenesulfonylhydrazone Derivatives of Methyl Dehydroabietate. Russ. J. Gen. Chem. 2019, 89, 819-823. [CrossRef]

17. Shaaban, M.M.; Ragab, H.M.; Akaji, K.; McGeary, R.P.; Bekhit, A.-E.A.; Hussein, W.M.; Kurz, J.L.; Elwakil, B.H.; Bekhit, S.A.; Ibrahim, T.M.; et al. Design, synthesis, biological evaluation and in silico studies of certain aryl sulfonyl hydrazones conjugated with 1,3-diaryl pyrazoles as potent metallo- $\beta$-lactamase inhibitors. Bioorg. Chem. 2020, 105, 104386. [CrossRef]

18. Loncle, C.; Brunel, J.M.; Vidal, N.; Dherbomez, M.; Letourneux, Y. Synthesis and antifungal activity of cholesterol-hydrazone derivatives. Eur. J. Med. Chem. 2004, 39, 1067-1071. [CrossRef]

19. Wang, H.; Ren, S.-X.; He, Z.-Y.; Wang, D.-L.; Yan, X.-N.; Feng, J.-T.; Zhang, X. Synthesis, Antifungal Activities and Qualitative Structure Activity Relationship of Carabrone Hydrazone Derivatives as Potential Antifungal Agents. Int. J. Mol. Sci. 2014, 15, 4257-4272. [CrossRef]

20. Backes, G.L.; Neumann, D.M.; Jursic, B.S. Synthesis and antifungal activity of substituted salicylaldehyde hydrazones, hydrazides and sulfohydrazides. Bioorg. Med. Chem. 2014, 22, 4629-4636. [CrossRef]

21. Gao, Z.; Lv, M.; Li, Q.; Xu, H. Synthesis of heterocycle-attached methylidenebenzenesulfonohydrazones as antifungal agents. Bioorg. Med. Chem. Lett. 2015, 25, 5092-5096. [CrossRef] 
22. Novakoski de Oliveira, K.; Costa, P.; Santin, J.R.; Mazzambani, L.; Bürger, C.; Mora, C.; Nunes, R.J.; de Souza, M.M. Synthesis and antidepressant-like activity evaluation of sulphonamides and sulphonyl-hydrazones. Bioorg. Med. Chem. 2011, 19, $4295-4306$. [CrossRef]

23. Abid, S.M.A.; Younus, H.A.; Al-Rashida, M.; Arshad, Z.; Maryum, T.; Gilani, M.A.; Alharthi, A.I.; Iqbal, J. Sulfonyl hydrazones derived from 3-formylchromone as non-selective inhibitors of MAO-A and MAO-B: Synthesis, molecular modeling and in-silico ADME evaluation. Bioorg. Chem. 2017, 75, 291-302. [CrossRef] [PubMed]

24. Aslan, H.G.; Özcan, S.; Karacan, N. Synthesis, characterization and antimicrobial activity of salicylaldehydebenzenesulfonylhydrazone (Hsalbsmh)and its Nickel(II), Palladium(II), Platinum(II), Copper(II), Cobalt(II) complexes. Inorg. Chem. Commun. 2011, 14, 1550-1553. [CrossRef]

25. Özdemir, Ü.Ö.; Arslan, F.; Hamurcu, F. Synthesis, characterization, antibacterial activities and carbonic anhydrase enzyme inhibitor effects of new arylsulfonylhydrazone and their Ni(II), Co(II) complexes. Spectrochim. Acta A 2010, 75, 121-126. [CrossRef] [PubMed]

26. Özdemir, Ü.Ö.; Akkaya, N.; Özbek, N. New nickel(II), palladium(II), platinum(II) complexes with aromatic methanesulfonylhydrazone based ligands. Synthesis, spectroscopic characterization and in vitro antibacterial evaluation. Inorg. Chim. Acta 2013, 400, 13-19. [CrossRef]

27. Özbek, N.; Özdemir, Ü.Ö.; Altun, A.F.; Şahin, E. Sulfonamide-derived hydrazone compounds and their Pd(II) complexes: Synthesis, spectroscopic characterization, X-ray structure determination, in vitro antibacterial activity and computational studies. J. Mol. Struct. 2019, 1196, 707-719. [CrossRef]

28. Çınarlı, M.; Çınarlı, E.; Ataol, Ç.Y.; İdil, O.; Kariptaş, E. Synthesis, structural characterization, Hirshfeld surface analysis, antimicrobial activity, and DNA cleavage studies of (Z)-4-methyl- $N^{\prime}$-(phenyl(pyridin-2-yl)methylene)benzenesulfonohydrazide and its $\mathrm{Co}(\mathrm{II}), \mathrm{Ni}(\mathrm{II})$ and $\mathrm{Zn}(\mathrm{II})$ complexes. J. Mol. Struct. 2019, 1196, 760-770. [CrossRef]

29. Özdemir, Ü.Ö.; Aktan, E.; Ilbiz, F.; Gündüzalp, A.B.; Özbek, N.; Sarı, M.; Çelik, Ö.; Saydam, S. Characterization, antibacterial, anticarbonic anhydrase II isoenzyme, anticancer, electrochemical and computational studies of sulfonic acid hydrazide derivative and its $\mathrm{Cu}(\mathrm{II})$ complex. Inorg. Chim. Acta 2014, 423, 194-203. [CrossRef]

30. Almendras, I.; Huentupil, Y.; Novoa, N.; Roussel, P.; Melis, D.R.; Smith, G.S.; Arancibia, R. Trinuclear Ni(II), Pd(II) and Cu(II) complexes containing the 2-hydroxybenzaldehyde-ferrocenyl-sulfonylhydrazone ligand: Synthesis, structural characterization and antiplasmodial evaluation. Inorg. Chim. Acta 2019, 496, 119050. [CrossRef]

31. Concha, C.; Quintana, C.; Klahn, A.H.; Artigas, V.; Fuentealba, M.; Biot, C.; Halloum, I.; Kremer, L.; Lopez, R.; Romanos, J.; et al. Organometallic tosyl hydrazones: Synthesis, characterization, crystal structures and in vitro evaluation of anti-Mycobecterium tuberculosis and antiproliferative activities. Polyhedron 2017, 131, 40-45. [CrossRef]

32. Popiołek, Ł.; Biernasiuk, A. Hydrazide-hydrazones of 3-methoxybenzoic acid and 4-tert-butylbenzoic acid with promising antibacterial activity against Bacillus spp. J. Enzym. Inhib. Med. Chem. 2016, 31 (Suppl. 1), 62-69. [CrossRef] [PubMed]

33. Popiołek, Ł.; Biernasiuk, A. Synthesis and investigation of antimicrobial activities of nitrofurazone analogues containing hydrazide-hydrazone moiety. Saudi Pharm. J. 2017, 25, 1097-1102. [CrossRef] [PubMed]

34. Popiołek, Ł.; Rysz, B.; Biernasiuk, A.; Wujec, M. Synthesis of promising antimicrobial agents: Hydrazide-hydrazones of 5nitrofuran-2-carboxylic acid. Chem. Biol. Drug Des. 2020, 95, 260-269. [CrossRef]

35. Popiołek, Ł.; Biernasiuk, A.; Malm, A. Synthesis and antimicrobial activity of new 1,3-thiazolidin-4-one derivatives obtained from carboxylic acid hydrazides. Phosphorus Sulfur Silicon Relat. Elem. 2015, 190, 251-260. [CrossRef]

36. Popiołek, Ł.; Patrejko, P.; Gawrońska-Grzywacz, M.; Biernasiuk, A.; Berecka-Rycerz, A.; Natorska-Chomicka, D.; PiątkowskaChmiel, I.; Gumieniczek, A.; Dudka, J.; Wujec, M. Synthesis and in vitro bioactivity study of new hydrazide-hydrazones of 5-bromo-2-iodobenzoic acid. Biomed. Pharm. 2020, 130, 110526. [CrossRef] [PubMed]

37. European Committee for Antimicrobial Susceptibility Testing (EUCAST). Determination of minimum inhibitory concentrations (MICs) of antibacterial agents by broth dilution. EUCAST Discussion document E. Dis 5.1. Clin. Microbiol. Infect. 2003, 9, 1-7.

38. Clinical and Laboratory Standards Institute. Reference Method for Broth Dilution Antifungal Susceptibility Testing of Yeasts; M27-S4; Clinical and Laboratory Standards Institute: Wayne, PA, USA, 2012. 